\title{
Third Law of Thermodynamics as a Single Inequality
}

\begin{abstract}
Henrik Wilming and Rodrigo Gallego
Dahlem Center for Complex Quantum Systems, Freie Universität Berlin, 14195 Berlin, Germany (Received 5 April 2017; revised manuscript received 1 July 2017; published 13 November 2017)

The third law of thermodynamics in the form of the unattainability principle states that exact ground-state cooling requires infinite resources. Here, we investigate the amount of nonequilibrium resources needed for approximate cooling. We consider as a resource any system out of equilibrium, allowing for resources beyond the independent and identically distributed assumption and including the input of work as a particular case. We establish in full generality a sufficient and a necessary condition for cooling and show that, for a vast class of nonequilibrium resources, these two conditions coincide, providing a single necessary and sufficient criterion. Such conditions are expressed in terms of a single function playing a role for the third law similar to the one of the free energy for the second law. From a technical point of view, we provide new results about the concavity or convexity of certain Renyi divergences, which might be of independent interest.
\end{abstract}

DOI: 10.1103/PhysRevX.7.041033

Subject Areas: Quantum Physics, Statistical Physics

\section{INTRODUCTION}

Pure quantum states are indispensable resources for any task in quantum information processing. However, the third law of thermodynamics (more precisely, the unattainability principle) states that cooling a system exactly to zero temperature requires an infinite amount of resources, whether it is in the form of time, space, work, or some other resource [1-5]. Similarly, no-go theorems have been put forward for the task of bit erasure-which is closely related to ground-state cooling-showing that no unitary process on a system and a finite-dimensional reservoir can bring the system from a mixed to a pure state [6-8]. However, these no-go results do not say much about the amount of resources needed for approximate cooling. Indeed, in recent times, a sizable number of studies dealt with different protocols to cool a small quantum system by unitarily acting on a heat bath and a certain number of systems out of equilibrium to be "used up" (known under the name of algorithmic or dynamical cooling) [9-13] or by studying particular models of refrigerating small quantum systems [14-20], including ones that seem to challenge the unattainability principle in terms of required time [21-28].

In this work, we focus on quantifying, in full generality, the expenditure of arbitrary systems out of equilibrium that are needed for approximate cooling while having access to a heat bath. Our scenario is similar to the one considered in algorithmic cooling, but here we treat the full thermodynamics of the problem by allowing for resources with

Published by the American Physical Society under the terms of the Creative Commons Attribution 4.0 International license. Further distribution of this work must maintain attribution to the author(s) and the published article's title, journal citation, and DOI. nontrivial Hamiltonians and accounting for the energy conservation of the total process. We do this in the resource theoretic framework of quantum thermodynamics [29-33], which has proven useful for answering a variety of fundamental questions in quantum thermodynamics, such as establishing an infinite family of second laws [34], providing fundamental bounds to single-shot thermodynamics [30,34-36], providing definitions of work for quantum systems $[30,37,38]$, generalizing fluctuation theorems $[39,40]$, elucidating the thermodynamic meaning of negative entropies [41], and elucidating the role of quantum coherence in thermodynamics [42-46].

Recently, there have also been studies on the problem of cooling from this point of view [3-5]; however, they mostly focused on providing necessary conditions in terms of resources such as time, space, or Hilbert-space dimension.

The task of cooling that we are considering can be phrased as finding a cooling protocol between an arbitrary resource described by the state and Hamiltonian $\rho_{R}$ and $H_{R}$, respectively, and a target system described by $\rho_{S}$ and $H_{S}$, where $\rho_{S}$ approximates the ground state of $H_{S}$. We later assume, for simplicity, that $\rho_{S}$ is a thermal state-in this case, the goal is to bring its final temperature $T_{S}$ to a very low value. We assume that the density matrix of the resource has full rank because otherwise the problem trivializes since one can, for example, simply swap with a ground state [47]. We furthermore assume that the target system is initially in thermal equilibrium with some environment. Then, the transition, i.e., the cooling protocol, can be performed by using a thermal bath at a fixed inverse temperature $\beta$ and performing a global unitary that commutes with the total Hamiltonian so that energy conservation is properly accounted for. These kinds of transitions have been extensively studied, and they can be characterized by families of functions $M^{\alpha}$, the so-called monotones, so that a transition is possible if and only if $[34,48,49]$ 


$$
M^{\alpha}\left(\rho_{R}, H_{R}\right) \geq M^{\alpha}\left(\rho_{S}, H_{S}\right) \quad \forall \alpha .
$$

Hence, the problem at hand is, in principle, hard to characterize since one needs to verify an infinite number of conditions to conclude that a given transition is possible. The main contribution of the present work is to show that, in the limit where $T_{S}$ is sufficiently close to zero-i.e., the regime where the (un)attainability problem is formulatedthe infinite set of monotones appearing in Eq. (10) can essentially be reduced to a single monotone. We call this monotone the vacancy, and it is defined as

$$
\mathcal{V}_{\beta}(\rho, H):=S\left(\omega_{\beta}(H) \| \rho\right),
$$

where $\omega_{\beta}(H)$ is the Gibbs state at inverse temperature $\beta$ and $S$ is the relative entropy defined as

$$
S(\rho \| \sigma)=\operatorname{Tr}(\rho \log \rho)-\operatorname{Tr}(\rho \log \sigma),
$$

if $\operatorname{supp}(\rho) \subseteq \operatorname{supp}(\sigma)$, and it is equal to $+\infty$ otherwise.

We find that sufficient and necessary conditions for cooling, respectively, are given by

$$
\begin{gathered}
\mathcal{V}_{\beta}\left(\rho_{R}, H_{R}\right)-K\left(\rho_{R}, H_{R}, \rho_{S}, H_{S}, \beta\right) \geq \mathcal{V}_{\beta}\left(\rho_{S}, H_{S}\right), \\
\mathcal{V}_{\beta}\left(\rho_{R}, H_{R}\right) \geq \mathcal{V}_{\beta}\left(\rho_{S}, H_{S}\right),
\end{gathered}
$$

where $K\left(\rho_{R}, H_{R}, \rho_{S}, H_{S}, \beta\right) \rightarrow 0$ as $T_{S} \rightarrow 0$. Hence, in the limit of very-low-temperature cooling, $\mathcal{V}_{\beta}\left(\rho_{S}, H_{S}\right)$ is the key quantity that determines the fundamental limitations. Importantly, $\mathcal{V}_{\beta}\left(\rho_{S}, H_{S}\right)$ diverges as $T_{S} \rightarrow 0$. The necessary condition (5) therefore shows that an infinite amount of resources (as measured by $\mathcal{V}_{\beta}$ ) is necessary for exact ground-state cooling. Furthermore, we show that for a vast class of resource systems-for example, thermal states of coupled harmonic oscillators-the function $K\left(\rho_{R}, H_{R}, \rho_{S}, H_{S}, \beta\right)$ vanishes identically. Hence,

$$
\mathcal{V}_{\beta}\left(\rho_{R}, H_{R}\right) \geq \mathcal{V}_{\beta}\left(\rho_{S}, H_{S}\right)
$$

becomes both a sufficient and necessary condition. That $\mathcal{V}_{\beta}$ plays an important role for the third law had previously been found in the setting of independent and identically distributed (IID) resources and qubits as target systems in the seminal work of Ref. [29]. Here, we extend the significance of the quantity $\mathcal{V}_{\beta}$ to arbitrary scenarios.

Usually, the unattainability principle is formulated with respect to time, arguing that an infinite amount of time (or infinitely many cycles of a periodically working machine) are needed to cool a system exactly to zero temperature. Our results show, for example, that if the nonequilibrium resources are simply hot thermal systems (as in the example of a thermal machine that operates between two heat baths), the system to be cooled and the cooling machine have to effectively interact with infinitely many such resource systems (or all parts of one infinitely large system). This implies that an infinite amount of time is needed since each such interaction takes a finite time (see Ref. [3] for a thorough discussion of this point).

Our findings not only serve to pose limitations to protocols of algorithmic cooling but also suggest a surprising symmetry between the second and third laws of thermodynamics. The second law-in its averaged version or in the version of the Jarzynski equality [50]— can be expressed in terms of the free-energy difference defined as

$$
\Delta F_{\beta}(\rho, H)=\frac{1}{\beta} S\left(\rho \| \omega_{\beta}(H)\right) .
$$

In analogy, we show that the third law can be expressed similarly in terms of $\mathcal{V}_{\beta}(\rho, H)$, which simply inverts the arguments of the relative entropy in Eq. (7) and drops the prefactor. This symmetry between the second and third laws is quite surprising and hints at the fact that the second and third laws can be related to the errors of the first and second kinds in hypothesis testing [51]. We leave the investigation of this deeper relation between the two for future work.

From a technical point of view, our results rely on certain convexity properties of the function $\alpha \mapsto S_{\alpha}(\rho \| \sigma)$, where $S_{\alpha}$ are classical Renyi divergences [51]. We believe that these results might be of independent interest.

\section{SETUP AND A GENERAL NECESSARY CONDITION}

In the following, we use the setup of catalytic thermal operations $[29,30,34]$ applied to the task of cooling. In this setup, we imagine possessing a resource given by a state and Hamiltonian pair $\left(\rho_{R}, H_{R}\right)$. We can then use an arbitrary thermal bath at inverse temperature $\beta$, that is, a system in a Gibbs state $\omega_{\beta}\left(H_{B}\right)$ of a Hamiltonian $H_{B}$, and finally an ancillary system, the so-called catalyst with an arbitrary state and a Hamiltonian $\left(\sigma_{C}, H_{C}\right)$, in such a way that the latter is returned in the same configuration and uncorrelated from the rest of the systems after implementing the protocol. The target system to be cooled is initially assumed to be in thermal equilibrium with the thermal bath and therefore described by a Gibbs state $\left(\omega_{\beta}\left(H_{S}\right), H_{S}\right)$. The total compound $R S B C$ is transformed by a cooling protocol, which consists simply of a unitary transformation $U$ that commutes with the total Hamiltonian.

More formally, we say that there exists a cooling protocol to $\rho_{S}$ using the resource $\left(\rho_{R}, H_{R}\right)$ if there exists a fixed catalyst $\left(\sigma_{C}, H_{C}\right)$, and for any $\epsilon>0$, there exists a unitary $U$ and a bath Hamiltonian $H_{B}$ such that

$$
\rho_{R S}^{\prime} \otimes \sigma_{C}^{\epsilon}=\operatorname{Tr}_{B}\left(U \rho_{R} \otimes \omega_{\beta}\left(H_{S}\right) \otimes \omega_{\beta}\left(H_{B}\right) \otimes \sigma_{C} U^{\dagger}\right)
$$


with $\operatorname{Tr}_{R}\left(\rho_{R S}^{\prime}\right)=\rho_{S}$ and $\left\|\sigma_{C}-\sigma_{C}^{\epsilon}\right\|_{1} \leq \epsilon$. The only constraint on the unitary $U$ is that it conserves the global energy, i.e.,

$$
\left[U, H_{R}+H_{S}+H_{B}+H_{C}\right]=0 .
$$

Note that this formulation of the cooling process contains, as a particular case, partial cooling in which we do not start with the target in a Gibbs state. In this case, the initial system of $S$, if it is partially cooled before starting the protocol, can be simply incorporated as part of the resource $R$.

The problem of finding conditions for the existence of a transitions of the form (8) has been studied in Ref. [34] for diagonal states, that is, with $\left[\rho_{R}, H_{R}\right]=0$ and $\left[\rho_{S}, H_{S}\right]=0$. Throughout this paper, we restrict ourselves to such diagonal states, but we emphasize that the necessary condition (5) also holds for nondiagonal states, as we will see later.

Under the assumption that $\rho_{R}$ and $\rho_{S}$ are diagonal, one can show that cooling to a state $\rho_{S}$ is possible if and only if [34]

$$
S_{\alpha}\left(\rho_{R} \| \omega_{\beta}\left(H_{R}\right)\right) \geq S_{\alpha}\left(\rho_{S} \| \omega_{\beta}\left(H_{S}\right)\right) \quad \forall \alpha \geq 0,
$$

where $S_{\alpha}$ are so-called Renyi divergences. The proof of this statement relies simply on the results of Ref. [34] together with the additivity of the Renyi divergences under tensor products.

An important tool that appears in Eq. (10) is the concept of a monotone of (catalytic) thermal operations [29,30]. This is any function $f$ that can only decrease under (catalytic) thermal operations. The functions $S_{\alpha}$ appearing in (10) are monotones under catalytic thermal operations and, more generally, under any channel that has the Gibbs state as a fixed point. Importantly, any monotone $f$, possibly different from $S_{\alpha}$, allows us to construct necessary conditions for a given transition. We now show that $\mathcal{V}_{\beta}$ is also a monotone under catalytic thermal operations, and we derive the corresponding necessary condition for cooling.

Theorem 1 (Monotonicity and necessary condition). The vacancy is an additive monotone under catalytic thermal operations. This has as an implication that for any target $\left(\rho_{S}, H_{S}\right)$ and resource $\left(\rho_{R}, H_{R}\right)$ —not necessarily diagonal states- the condition

$$
\mathcal{V}_{\beta}\left(\rho_{R}, H_{R}\right) \geq \mathcal{V}_{\beta}\left(\rho_{S}, H_{S}\right)
$$

is necessary for cooling.

Proof.-Let us first show that $\mathcal{V}_{\beta}$ is a monotone under catalytic thermal operations. Let us consider an arbitrary transition from state $\rho$ to state $\rho^{\prime}$-both with Hamiltonian $\mathrm{H}$-by catalytic thermal operations; then, we show that $\mathcal{V}_{\beta}(\rho, H) \geq \mathcal{V}_{\beta}\left(\rho^{\prime}, H\right)$.
First, note that the vacancy diverges for a state $\rho$ without full rank; thus, the inequality $\mathcal{V}_{\beta}(\rho, H) \geq \mathcal{V}_{\beta}\left(\rho^{\prime}, H\right)$ is satisfied trivially for those states. Let us therefore assume that $\rho$ is a full-rank state. As was shown in Ref. [34], for any $0 \leq \alpha \leq 2$, the Renyi divergences

$$
S_{\alpha}\left(\rho \| \omega_{\beta}(H)\right):=\frac{1}{\alpha-1} \log \operatorname{Tr}\left(\rho^{\alpha} \omega_{\beta}(H)^{1-\alpha}\right)
$$

are monotonic under (catalytic) thermal operations for arbitrary states $\rho$. In other words, we have the necessary condition

$$
S_{\alpha}\left(\rho \| \omega_{\beta}(H)\right) \geq S_{\alpha}\left(\rho^{\prime} \| \omega_{\beta}(H)\right) \quad \forall 0 \leq \alpha \leq 2,
$$

By simple algebra, one can show that

$$
\left.\frac{\partial}{\partial_{\alpha}}\right|_{\alpha=0} S_{\alpha}\left(\rho \| \omega_{\beta}(H)\right)=V_{\beta}(\rho, H) \geq 0 .
$$

Taylor expanding Eq, (13) on both sides for any $\alpha>0$ and dividing by $\alpha$ then yields

$$
V_{\beta}(\rho, H)+O(\alpha) \geq V_{\beta}\left(\rho^{\prime}, H\right)+O(\alpha),
$$

where $O(\alpha)$ indicates that it is of first order in $\alpha$. Taking $\alpha$ arbitrarily small then yields $V_{\beta}(\rho, H) \geq V_{\beta}\left(\rho^{\prime}, H\right)$. This proves monotonicity under catalytic thermal operations. Additivity follows directly from the additivity of the relative entropy under tensor products.

Since we established that the vacancy is a monotone under catalytic thermal operations, we can now derive the necessary condition for cooling by simply applying this condition to the transition that $S R$ undergo in the cooling process:

$$
\begin{aligned}
& \mathcal{V}_{\beta}\left(\rho_{R} \otimes \omega_{\beta}\left(H_{S}\right), H_{R}+H_{S}\right) \\
& \quad \geq \mathcal{V}_{\beta}\left(\rho_{R S}^{\prime}, H_{R}+H_{S}\right) \\
& \quad \geq \mathcal{V}_{\beta}\left(\omega_{\beta}\left(H_{R}\right) \otimes \rho_{S}, H_{R}+H_{S}\right) .
\end{aligned}
$$

The last inequality follows from the fact that one can always replace the state on any system by an uncorrelated thermal state at the heat bath's temperature using a thermal operation. Using additivity of the vacancy and the fact that $\mathcal{V}_{\beta}\left(\omega_{\beta}(H), H\right)=0$, we obtain the necessary condition (11)

We emphasize that the necessary condition (11) is derived in full generality, and it applies to any full-rank state $\rho_{R}$ and any state $\rho_{S}$, possibly not diagonal in the eigenbasis of $H_{S}$.

The monotone $\mathcal{V}_{\beta}$ was first introduced in Ref. [29]. Its relevance for the unattainability principle is clear since if $\rho_{S}$ does not have full support, then the right-hand side of Eq. (11) diverges. Hence, exact cooling is impossible 
unless the resource $\rho_{R}$ does not have full support either. In the particular case of $\rho_{R}=\bigotimes_{i=1}^{n} Q^{i}$ where $\varrho^{i}$ has full support, the condition (11) already tells us that we need infinite resources-infinite $n$ in this case-for exact cooling. Hence, such a simple analysis already suggests that the quantity $\mathcal{V}_{\beta}$ plays a crucial role for the limitations on cooling.

To summarize, we have seen, building upon previous literature, that the vacancy $\mathcal{V}_{\beta}$ establishes completely general necessary conditions for cooling. However, for necessary and sufficient conditions, one should, in principle, verify an infinite number of inequalities given by Eq. (10). Our contribution will be to show that these infinite numbers of inequalities can be reduced to a single one, which can also be expressed in terms of the vacancy for a sufficiently cold final state $\rho_{S}$. Furthermore, we show that for a large family of resource systems, the single sufficient condition that we find coincides with the necessary condition (11). Hence, the limits on cooling are entirely ruled by the function $\mathcal{V}_{\beta}$. This holds for large classes of finite systems, with possibly correlated and interacting subsystems.

\section{GENERAL SUFFICIENT CONDITIONS FOR COOLING}

The process of cooling laid out in the previous section can, in principle, be applied to any final state $\rho_{S}$. We now assume, for simplicity, that the final state, as it corresponds to a cooling process, is of the form $\rho_{S}=\omega_{\beta_{S}}\left(H_{S}\right)$ with $\beta_{S}$ very large. We can then derive the following completely general sufficient condition for cooling.

Theorem 2 (General sufficient condition for cooling). For every choice of $\beta$ and $H_{S}$, there is a critical $\beta_{\text {cr }}>0$ such that, for any $\beta_{S}>\beta_{\text {cr }}$ and full-rank resource $\left(\rho_{R}, H_{R}\right)$, the condition

$$
\mathcal{V}_{\beta}\left(\rho_{R}, H_{R}\right)-K\left(\beta_{S}, \beta, \rho_{R}, H_{R}, H_{S}\right) \geq \mathcal{V}_{\beta}\left(\omega_{\beta_{S}}\left(H_{S}\right), H_{S}\right)
$$

is sufficient for cooling. The positive semidefinite function $K$ has the property $K\left(\beta_{S}, \beta, \rho_{R}, H_{R}\right) \rightarrow 0$ as $\beta_{S} \rightarrow \infty$ for any fixed $\beta, H_{R}, \rho_{R}>0$ and $H_{S}$.

The proof of the theorem is given in Sec. VI. Nonetheless, we provide a sketch of the main ideas involved in such a proof at the end of this section. The function $K$ is given by

$$
\begin{aligned}
& K\left(\beta_{S}, \beta, \rho_{R}, H_{R}, H_{S}\right) \\
& \quad=\max \left\{0,-\delta\left(\beta_{S}\right) \min _{\alpha \leq \delta\left(\beta_{S}\right)} \frac{\partial^{2}}{\partial \alpha^{2}} S_{\alpha}\left(\rho_{R} \| \omega_{\beta}\left(H_{R}\right)\right)\right\},
\end{aligned}
$$

where

$$
\delta\left(\beta_{S}\right):=\log \left(Z_{\beta}\right) / \mathcal{V}_{\beta}\left(\omega_{\beta_{S}}\left(H_{S}\right), H_{S}\right) \geq 0, \quad Z_{\beta}=\operatorname{Tr}\left(\mathrm{e}^{-\beta H_{S}}\right) .
$$

The bound (16) applies for any possible (diagonal) resource state; however, finding $K\left(\beta_{S}, \beta, \rho_{R}, H_{R}, H_{S}\right)$ involves a minimization, which, although feasible for lowdimensional systems, might be an obstacle for practical purposes when dealing with large systems and for values of $\beta_{S}$, so $K\left(\beta_{S}, \beta, \rho_{R}, H_{R}, H_{S}\right)$ cannot be neglected. That said, we will investigate resource systems $\rho_{R}, H_{R}$ for which $K\left(\beta_{S}, \beta, \rho_{R}, H_{R}, H_{S}\right)=0$. In those cases, the general sufficient condition given by Eq. (16), taken together with the necessary condition (6), will imply that a necessary and sufficient condition is given simply by

$$
\mathcal{V}_{\beta}\left(\rho_{R}, H_{R}\right) \geq \mathcal{V}_{\beta}\left(\omega_{\beta_{S}}\left(H_{S}\right), H_{S}\right) .
$$

In particular, we see in Sec. V that this holds true for large classes of thermal nonequilibrium resources. Let us also note that $K\left(\beta_{S}, \beta, \rho_{R}, H_{R}, H_{S}\right)$, just as the vacancy, is additive over noninteracting and uncorrelated resources. We use this property in the next section to investigate the setting of IID resources.

In the result given above, we have focused on thermal target states. This is, in fact, unnecessary. We show in Appendix E that a completely analogous result holds for states of the form

$$
\rho_{\epsilon}=(1-\epsilon)|0\rangle\langle 0|+\epsilon \rho^{\perp}, \quad \epsilon \ll 1,
$$

where $\rho^{\perp}$ is any density matrix that has full rank on the subspace orthogonal to the ground state $|0\rangle$ and commutes with $H_{S}$.

\section{A. Sketch of the proof of Theorem 2}

As we have seen in the previous section, a set of sufficient conditions for a transition with catalytic thermal operations is given by the infinite set of inequalities of Eq. (10). The main idea behind the proof is that when the target system is sufficiently cold $\left(\beta_{S}>\beta_{\text {cr }}\right)$, it suffices to check the conditions (10) for very small $\alpha$. This follows from the fact that for $\beta_{S}>\beta_{\text {cr }}$, the right-hand side of Eq. (10), given by $S_{\alpha}\left(\rho_{S} \| \omega_{\beta}\left(H_{S}\right)\right)$, rapidly saturates to its maximum value as we increase $\alpha$, and it is concave (see Fig. 1). Given that one only needs to consider small values of $\alpha$, it is possible to make a Taylor expansion around $\alpha=0$ of $S_{\alpha}$ of the form

$$
\begin{aligned}
S_{\alpha}\left(\rho_{R} \| \omega_{\beta}\left(H_{R}\right)\right) \approx & S_{0}\left(\rho \| \omega_{\beta}\left(H_{R}\right)\right)+\left.\frac{\partial S_{\alpha}\left(\rho \| \omega_{\beta}\left(H_{R}\right)\right)}{\partial \alpha}\right|_{\alpha=0} \alpha \\
& +k \alpha^{2} .
\end{aligned}
$$

This reduces the infinite inequalities of Eq. (1) to a single one that depends on the derivate of $S_{\alpha}$ and an error term $k$, which is related to the error term appearing in Theorem 2 


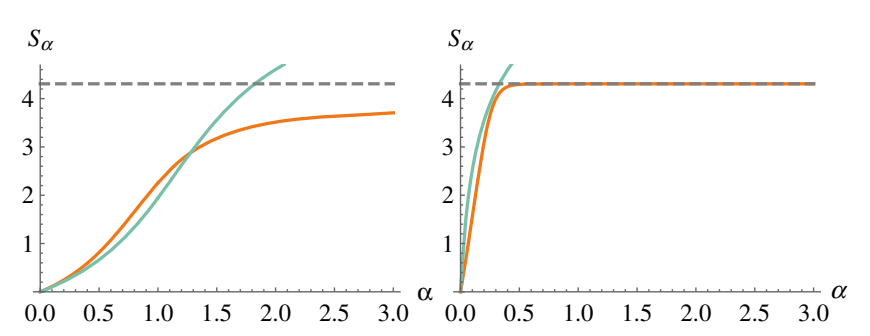

FIG. 1. The figure shows the behavior of $S_{\alpha}\left(\omega_{\beta_{S}}\left(H_{S}\right) \| \omega_{\beta}\left(H_{S}\right)\right)$ (orange line) and $S_{\alpha}\left(\rho_{R} \| \omega_{\beta}\left(H_{R}\right)\right)$ (blue line). The left plot shows a target state that is not very cold, together with an insufficient resource. The transition is not possible since the blue line is below the orange line for $\alpha \lesssim 1.25$. The right plot shows the behavior when $\rho_{S}$ becomes very cold. The function becomes more similar to a step function. The fact that $S_{\alpha}\left(\rho_{R} \| \omega_{\beta}\left(H_{R}\right)\right)$ (blue curve) is larger than the orange curve, which implies that the transition is possible, is determined by the behavior at very small values of $\alpha$, and up to a small error, by the fact that the derivate of the blue curve is larger than that of the orange curve at $\alpha=0$.

denoted by $K$. This expansion can be further simplified by noting that $S_{\alpha=0}\left(\rho \| \omega_{\beta}(H)\right)=0$. The vacancy comes into play because of the identity

$$
\left.\frac{\partial S_{\alpha}(\rho \| \sigma)}{\partial \alpha}\right|_{\alpha=0}=S_{1}(\sigma \| \rho):=S(\sigma \| \rho)
$$

which inverts the arguments of the second term on the right-hand side of Eq. (19). Taking all these elements into account and accounting properly for the precision of the Taylor approximations, we arrive at an inequality involving only the vacancy and a vanishing error term as determined by Theorem 2 .

\section{B. Short comment on catalysts}

As laid out in Sec. II, we define the catalytic thermal operations by including the possibility that the catalyst changes during the transition, as long as this change can be made arbitrarily small, as is standard in recent literature on the resource theoretic approach to thermodynamics [34]. This formulation is a form of exact catalysis, in the sense that as the error has to be arbitrarily small, the catalyst is returned arbitrarily unchanged. However, it is possible to consider other forms of catalytic thermal operations that are either more restrictive about the change of the catalyst (where no error-not even arbitrarily small-is allowed for) or less restrictive, in the sense that the catalyst is allowed to change by a finite amount. We consider both alternatives in Appendixes F and G, respectively. First, we study the case in which one requires that the catalyst is always returned exactly in the same state, that is, taking $\epsilon=0$ in the definitions laid out in Sec. II. In this case, it is no longer valid that a set of sufficient conditions is given by positive values of $\alpha$ in Eq. (10), but one also has to consider Renyi divergences for negative $\alpha$. This case is analyzed in
Appendix F, where we show that, in this scenario, (i) the general necessary condition of Theorem 2 holds and (ii) a general sufficient condition similar to the one of Theorem 2 is derived. This general sufficient condition only differs on a multiplicative constant-independent of the final temperature to which one cools-from the one derived in Theorem 2. Finally, in Appendix G, we furthermore discuss the case of approximate catalysts. We put forward a consistent method to allow for finite errors on the catalyst while maintaining the validity of the third law of thermodynamics.

\section{I.I.D RESOURCES AND SCALING OF THE TARGET TEMPERATURE}

Theorem 2, together with the necessary condition (11), provides completely general sufficient and necessary conditions, respectively, for cooling a system to target temperature $T_{S}=1 / \beta_{S}$ (setting $k_{B}=1$ ) using a given resource $\left(\rho_{R}, H_{R}\right)$. Thus, they characterize the possibility of cooling in full generality. To obtain results for concrete physical situations and find out how the target temperature $T_{S}$ scales with physical key quantities of the resource - such as the system size of the resource-one has to choose a particular resource and calculate its vacancy as well as the error term $K$. Then, one has to check how these quantities depend on the physical properties of interest.

We now focus on the scaling between the size of the resource and the final temperature of the target system. We assume that the resource is given by a number of identically and independently distributed copies. Later, we also discuss other assumptions we can make about the resource. Thus, we consider the case where the resource state is given by $\rho_{R}=\varrho_{R}^{\otimes n}$ and Hamiltonian $H_{R}=\sum_{i} H_{R}^{i}$,

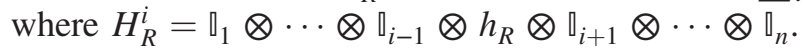

Let us now consider the following task: Given fixed $\varrho_{R}$, $H_{R}, \beta, H_{S}$, find the minimum $n$ so that it is possible to cool down the target state to inverse temperature $\beta_{S}$.

By using Theorem 1, together with the additivity of $\mathcal{V}_{\beta}$, we obtain that the necessary number of copies $n^{\text {nec }}\left(\beta_{S}\right)$ fulfills

$$
n^{\mathrm{nec}}\left(\beta_{S}\right) \geq \frac{\mathcal{V}_{\beta}\left(\omega_{\beta_{S}}\left(H_{S}\right), H_{S}\right)}{\mathcal{V}_{\beta}\left(\varrho_{R}, h_{R}\right)} .
$$

By using Theorem 2 in the IID, we also obtain a sufficient number of copies $n^{\text {suff }}$. The condition (F2) takes the form

$$
n\left[\mathcal{V}_{\beta}\left(\varrho_{R}, h_{R}\right)-K\left(\beta_{S}, \beta, \varrho_{R}, h_{R}, H_{S}\right)\right] \geq \mathcal{V}_{\beta}\left(\omega_{\beta_{S}}\left(H_{S}\right), H_{S}\right)
$$

Since this condition is sufficient, but not always necessary, we obtain

$$
n^{\text {suff }}\left(\beta_{S}\right) \leq \frac{\mathcal{V}_{\beta}\left(\omega_{\beta_{S}}\left(H_{S}\right), H_{S}\right)}{\mathcal{V}_{\beta}\left(\varrho_{R}, h_{R}\right)-K\left(\beta_{S}, \beta, \varrho_{R}, h_{R}, H_{S}\right)} .
$$


Since the correction $K$ goes to zero as $\beta_{S} \rightarrow \infty$ (with the target temperature going to zero), we see that

$$
\lim _{\beta_{S} \rightarrow \infty} \frac{n^{\text {suff }}\left(\beta_{S}\right)}{n^{\text {nec }}\left(\beta_{S}\right)}=1
$$

It is also interesting to reexpress the previous conditions to obtain a more transparent relation between the final achievable temperature and the number of copies. We see next that $n^{\text {nec }}\left(\beta_{S}\right)$ and $n^{\text {suff }}\left(\beta_{S}\right)$ scale as $\beta_{S}$ for large $\beta_{S}$. Thus, the target temperature approaches zero as $1 / n$.

\section{A. Scaling of the target temperature}

In the special case where the target state is a thermal state, one can reformulate the vacancy in terms of nonequilibrium free energies. Indeed, the vacancy of a thermal state at temperature $\beta_{S}$ simply takes the form

$$
\mathcal{V}_{\beta}\left(\omega_{\beta_{S}}(H), H\right)=\beta_{S} \Delta F_{\beta_{S}}\left(\omega_{\beta}\left(H_{S}\right), H_{S}\right),
$$

with $\Delta F_{\beta}(\rho, H)=\langle H\rangle_{\rho}-\langle H\rangle_{\beta}-\left(S(\rho)-S\left(\omega_{\beta}\right)\right) / \beta$.

In this case, the condition (11) reads

$$
\mathcal{V}_{\beta}\left(\rho_{R}, H_{R}\right) \geq \beta_{S} \Delta F_{\beta_{S}}\left(\omega_{\beta}\left(H_{S}\right), H_{S}\right) .
$$

From Eq. (24), we see that for large $\beta_{S}$, we have (assuming vanishing ground-state energy)

$$
\mathcal{V}_{\beta}\left(\omega_{\beta_{S}}(H), H\right)=\beta_{S} E_{\beta}-S_{\beta}, \quad \text { as } \quad \beta_{S} \rightarrow \infty .
$$

Assuming again a resource system of $n$ noninteracting identical particles each described by $\left(\varrho_{R}, h_{R}\right)$, we then obtain that the minimum achievable temperature $T_{S}^{(n)}$ scales as

$$
T_{S}^{(n)}=\frac{1}{n} \frac{E_{\beta}^{S}}{\mathcal{V}_{\beta}\left(\varrho_{R}, h_{R}\right)}, \quad n \gg 1 .
$$

This result is similar to the asymptotic result of Janzing et al. [29].

Lastly, let us point out that the above scaling relation implies that the probability $p$ to find the system in the ground state after the cooling procedure increases exponentially to 1 with $n$. For example, if the target system is a $d+1$-dimensional system with gap $\Delta$ above a unique ground state, we have (for large $n$ )

$$
p \geq 1 /\left(1+d \mathrm{e}^{-\beta_{S} \Delta}\right) \approx 1-d \mathrm{e}^{-n \mathcal{V}_{\beta}\left(\omega_{\beta_{R}}\left(h_{R}\right), h_{R}\right)\left[(\Delta) / E_{\beta}^{S}\right]} .
$$

Thus, while an exact third law holds in the sense that $n \rightarrow \infty$ for $T_{S} \rightarrow 0$, the ground-state probability asymptotically converges very quickly to unity.

The above relations demonstrate how one can obtain quantitative expressions of the unattainability principle from Theorems 1 and 2 by making assumptions about the given resources.

\section{B. Scaling of the vacancy with system size}

In the case of IID resources with noninteracting Hamiltonians, the vacancy is an extensive quantity in the sense that it scales linearly with the number of particles. However, for arbitrary quantum systems with correlated and interacting constituents, it is, in general, difficult to calculate the vacancy and hence estimate directly how it scales with the number of particles. Nonetheless, we can use the relation (24) to argue that the vacancy will be extensive for large classes of many-body systems.

In particular, let us assume that the resource is in a thermal state of some local Hamiltonian. In other words, $\rho_{R}=\omega_{\tilde{\beta}}\left(\tilde{H}_{R}\right)$, where $\tilde{H}_{R}$ is any local Hamiltonian (possibly differing from $H_{R}$ ) and $\tilde{\beta}$ is finite. In this case, one can use Eq. (24) to write

$$
\mathcal{V}_{\beta}\left(\rho_{R}, H_{R}\right)=\tilde{\beta} \Delta F_{\tilde{\beta}}\left(\omega_{\beta_{R}}\left(H_{R}\right), \tilde{H}_{R}\right) .
$$

From the fact that the von Neumann entropy is subadditive, and from the locality of the Hamiltonians $H_{R}$ and $\tilde{H}_{R}$, it then follows that the vacancy $\mathcal{V}_{\beta}\left(\rho_{R}, H_{R}\right)$ scales (at most) linearly with the system size. As a consequence, the minimal final temperature $T_{S}^{(n)}$ scales (at best) inversely proportional to the volume of the resource.

In light of the previous considerations, it seems likely that a similar scaling holds for any resource (potentially under reasonable physical assumptions, such as clustering of correlations). We leave the general characterization of many-body systems such that the vacancy is extensive as an interesting future research direction.

\section{THERMAL RESOURCES}

As discussed after the statement of Theorem 2, it is useful to find general conditions under which the error term $K$ disappears and the sufficient condition coincides with the general necessary condition. Naturally, it is necessary to make additional assumptions about the resources to achieve this.

We now consider as resource state $\rho_{R}$ a (possibly multipartite) thermal state of some Hamiltonian $H_{R}$ at inverse temperature $\beta_{R}$. In the following, we derive a simple expression that allows us to check whether

$$
K\left(\beta_{S}, \beta, \rho_{R}, H_{R}, H_{S}\right)=0, \quad \forall \beta_{S}>0, H_{S},
$$

and hence, Eq. (11) becomes a necessary and sufficient condition. The reasoning is based on showing that

$$
S_{\alpha}\left(\rho_{R}=\omega_{\beta_{R}}\left(H_{R}\right) \| \omega_{\beta}\left(H_{R}\right)\right)
$$


is convex for a given range of $\alpha<1$, which implies Eq. (29). The convexity of Eq. (30) can be determined by looking at the convexity of the average energy as a function of the inverse temperature of the resource,

$$
x \mapsto E_{x}^{R}:=\operatorname{Tr}\left(\omega_{x}\left(H_{R}\right) H_{R}\right) .
$$

In particular, we see that if $\beta_{R}<\beta$ and the function $x \mapsto E_{x}^{R}$ is convex for $x \in\left[\beta_{R}, \beta\right]$, then the function $S_{\alpha}\left(\omega_{\beta_{R}}\left(H_{R}\right) \| \omega_{\beta}\left(H_{R}\right)\right)$ is convex for all $\alpha<1$.

Theorem 3. For resources of the form $\left(\omega_{\beta_{R}}\left(H_{R}\right), H_{R}\right)$ that are hotter than the bath, that is, with $\beta_{R} \leq \beta$, if $E_{x}^{R}=$ $\operatorname{Tr}\left(\omega_{x}\left(H_{R}\right) H_{R}\right)$ is convex in the range $x \in\left[\beta_{R}, \beta\right]$, then Eq. (11) is a sufficient and necessary condition for lowtemperature cooling.

This theorem considerably simplifies the task of formulating bounds on the third law since the average energy is a much more accessible quantity than the Renyi divergences. In Sec. VA, we discuss several classes of physically motivated conditions that imply that $E_{x}^{R}=$ $\operatorname{Tr}\left(\omega_{x}\left(H_{R}\right) H_{R}\right)$ is convex. We emphasize, however, that the convexity of the energy is not a necessary condition for the correction $K$ to vanish: There are cases for which $x \mapsto E_{x}^{R}$ is not convex for the whole range of inverse temperatures $\left[\beta_{R}, \beta\right]$, and Eq. (11) is nevertheless a sufficient and necessary condition for cooling.

Lastly, let us mention that condition (29) is fulfilled if, for a fixed $\beta_{R}$, the bath's inverse temperature $\beta$ is sufficiently large, without any extra assumption on the convexity of $E_{x}$. This implies that, for sufficiently cold baths, Eq. (11) is also a sufficient and necessary condition. This is shown in Appendix B, together with several properties of the Renyi divergences for thermal states that might be of independent interest and also include a proof of Theorem 3.

\section{A. Systems for which the energy is convex}

As implied by Theorem 3, Eq. (11) becomes a sufficient and necessary condition for cooling if the resource is a thermal state hotter than the bath and its average energy is convex in the inverse temperature. We now see that the convexity of the energy is fulfilled by a wide range of physical models.

We first reexpress the convexity in terms of the heat capacity. This allows us to check whether $E_{x}^{R}$ is convex for a vast family of systems, as the heat capacity as a function of the temperature is an intensively studied quantity for many-body systems. Using the definition of heat capacity $C_{x}:=\left[\left(\mathrm{d} E_{x}^{R}\right) / \mathrm{d} T\right]$, with $T=1 / x$, we find that the convexity of the energy, as formulated in the condition of Theorem 3, can be expressed as

$\frac{\mathrm{d}^{2} E_{x}^{R}}{\mathrm{~d} x^{2}}=\frac{1}{x^{2}}\left(\frac{C_{x}}{x}-\frac{\mathrm{d} C_{x}}{\mathrm{~d} x}\right) \geq 0 \quad$ with $\quad x \in\left[\beta_{R}, \beta\right]$.

Equivalently, this condition can be expressed as

$$
\frac{1}{\beta_{R}^{2}} C_{\beta_{R}}-\frac{1}{\beta^{\prime 2}} C_{\beta^{\prime}} \geq 0
$$

for all $\beta_{R} \leq \beta^{\prime} \leq \beta$. In most thermodynamics systems, the heat capacity is monotonically increasing with the temperature; hence, $\left[\left(\mathrm{d} C_{x}\right) / \mathrm{d} x\right] \leq 0$ and Eq. (32) is satisfied. A seminal exception to this case is given by the so-called Schottky anomaly, which is present in certain solid states at very low temperatures [52]. We thus see that for thermodynamic systems, the convexity of the energy is a very natural property. Nevertheless, it can fail-in particular, in finite systems. We now show that even for large classes of finite systems, the energy is convex because of the following lemma, which we prove in the Appendix C.

Lemma 4 (Equidistant levels). Consider any Hamiltonian with equidistant and nondegenerate energy levels. Then, the function $\beta \mapsto E_{\beta}$ is convex.

Immediate examples of Hamiltonians with equidistant energy levels are two-level systems or harmonic oscillators. But in fact, the lemma covers a much wider class of models since the vacancy is unitarily invariant and additive over noninteracting subsystems.

It follows that any harmonic system and any system described by free fermions also have a convex energy function since free bosonic and fermionic systems can always be made noninteracting by a normal-mode decomposition. In such a normal-mode decomposition, they simply correspond to a collection of noninteracting harmonic oscillators or two-level systems, respectively.

These systems include highly correlated (even entangled) systems, and no thermodynamic limit needs to be taken. A particularly interesting resource that is included in these results is that of hot thermal light, which has been considered before as a valuable resource for cooling [18].

It can furthermore be checked that for large but finite many-body systems whose density of states in the bulk is well approximated by $\mu(\epsilon) \simeq \mathrm{e}^{\gamma \epsilon-\alpha \epsilon^{2}}$, the average energy $E_{\beta}$ is convex in $\beta$ [53].

Finally, for every finite system, there is a critical $\beta_{c}$ such that $E_{\beta}$ is convex for all $\beta>\beta_{c}$. Thus, as soon as $\beta>\beta_{R}>\beta_{c}$, the sufficient condition (11) holds for small enough target temperatures. This means that if an experimenter has a mechanism to precool the environment to a very low temperature, well below $1 / \beta_{c}$, and the resource has a temperature larger than that of the environment but still smaller than $1 / \beta_{c}$, then condition (11) holds as a sufficient and necessary condition.

\section{B. Source of work}

Our formalism can also incorporate a source of work as a particular case of a resource for cooling. The limitations on cooling as a function of the input of work have been studied in Ref. [3]. There, it is shown that the fluctuations of work, rather than its average value, have to diverge when the 
target state reaches vanishing final temperature and if the heat bath has finite heat capacity. Here, we derive a result similar in spirit using Theorem 2, although we employ a different model for the work source. Importantly, our result implicitly allows for infinite heat capacity in the heat bath. It should thus be viewed as being complementary to the results in Ref. [3].

Let us model the work source by a system $R$ with Hamiltonian

$$
H_{R}^{w}=\sum_{x=-d / 2}^{d / 2} \frac{E x}{d}\left|\frac{E x}{d}\right\rangle\left\langle\frac{E x}{d}\right| .
$$

One can see this Hamiltonian as a $d$-dimensional harmonic oscillator with energies bounded between $E / 2$ and $-E / 2$. We are interested in the limit of $d \rightarrow \infty$. In this case, $R$ is similar to the model put forward in Refs. [3,16], with the difference that here we consider a finite value of $E$. We enforce the condition that the battery acts as a energy reservoir and not as an entropy sink (which would make the task of cooling trivial) by assuming the work source to be in state $\rho_{R}^{w}=\square / d$ (we can also interpret this as the work source being at temperature $+\infty$ ). These assumptions on the work source are justified by the fact that it fulfills the second law of thermodynamics.

In other words, suppose we want to use a nonequilibrium state $\rho$ of some system $S$ with Hamiltonian $H_{S}$ to extract work and put it into the work source as the average energy. To do this, we implement a (catalytic) thermal operation on the heat bath, system $S$, and the work source $R$. Then, the increase of energy on the work source (i.e., the work) $\Delta E_{R}$ is bounded by the nonequilibrium free energy of the system as

$$
\Delta E_{R} \leq \Delta F_{\beta}\left(\rho, H_{S}\right)
$$

This is shown in Appendix D.

Now, we show that the third law can be obtained, in the sense that both $E$ and $d$ have to diverge in order to be able to use $R$ to cool down a system to zero final temperature. Let us first recall that by Lemma 4 , a sufficient and necessary condition for cooling for such a resource is given by Eq. (11). Furthermore, the vacancy of the work source is given by

$$
\begin{aligned}
\mathcal{V}_{\beta}\left(\rho_{R}^{w}, H_{R}^{w}\right) & =S\left(\omega_{\beta}\left(H_{R}^{w}\right) \| \llbracket / d\right) \\
& =\operatorname{Tr}\left(\omega_{\beta}\left(H_{R}^{w}\right)\left[\log \left(\omega_{\beta}\left(H_{R}^{w}\right)-\log (\llbracket / d)\right)\right]\right) \\
& =-\beta \operatorname{Tr}\left(\omega_{\beta}\left(H_{R}^{w}\right) H_{R}^{w}\right)+\log (d)-\log \left(Z_{\beta}\left(H_{R}^{w}\right)\right) \\
& \leq-\beta E / 2+\log (d)-\log \left(Z_{\beta}\left(H_{R}^{w}\right)\right) .
\end{aligned}
$$

The partition function can be upper bounded as

$$
\begin{aligned}
Z_{\beta}\left(H_{R}^{w}\right) & =\sum_{x=-d / 2}^{d / 2} e^{-\beta[(E x) / d]} \\
& \geq e^{\beta E / 2}+(d-1) e^{-\beta E / 2} \\
& =e^{\beta E / 2} d\left(\frac{1}{d}+\frac{d-1}{d} e^{-\beta E}\right) .
\end{aligned}
$$

Hence, we find that

$$
\mathcal{V}_{\beta}\left(\rho_{R}^{w}, H_{R}^{w}\right) \leq-\log \left(\frac{1}{d}+\frac{d-1}{d} e^{-\beta E}\right) .
$$

Combined with Eq. (11), this implies that a necessary condition for cooling to a state $\rho_{S}$ is given by

$$
\mathcal{V}_{\beta}\left(\rho_{S}, H_{S}\right) \leq-\log \left(\frac{1}{d}+\frac{d-1}{d} e^{-\beta E}\right) .
$$

Most importantly, note that in order to obtain a state $\rho_{S}$ that is close to a Gibbs state at zero temperature, the right-hand side of Eq. (41) has to diverge. For this to be possible, both $E$ and $d$ have to diverge since

$$
\begin{gathered}
\lim _{d \rightarrow \infty} \mathcal{V}_{\beta}\left(\rho_{R}^{w}, H_{R}^{w}\right) \leq \beta E, \\
\lim _{E \rightarrow \infty} \mathcal{V}_{\beta}\left(\rho_{R}^{w}, H_{R}^{w}\right) \leq \log (d) .
\end{gathered}
$$

This implies the unattainability principle, in the sense that an infinitely dense spectrum with unbounded energy is needed for cooling to absolute zero.

Before coming to the proof of Theorem 2 and our conclusions, let us briefly comment on a different model of work and a possible source of confusion that might arise. A model of work known as a work bit has been used in the literature of thermal operations [30,34]. In this model, it is assumed that the work source is a two-level system with energy gap $W$ that undergoes a transition from the excited state $|W\rangle$ to the ground state $|0\rangle$ to implement a transition on system $S$. Using the results of Ref. [30], one can show that it is possible to cool a system to the ground state in this model as long as $W>\log Z_{\beta}$, where $Z_{\beta}$ is the partition function of the system $S$. This means that there exists a (catalytic) thermal operation that implements cooling, in the sense that

$$
\omega_{\beta}\left(H_{S}\right) \otimes|W\rangle\langle W|\mapsto| 0\rangle\langle 0|\otimes| 0\rangle\langle 0| .
$$

At first, this seems to be in conflict with our results. However, using the vacancy, it is easy to show that the above process is extremely unstable: It only works for pure initial states of the work bit. Indeed, one can use condition (11) to establish limits on cooling if the initial state on the work bit is any full-rank state that approximates the excited 
state $|W\rangle\langle W|$ to arbitrary but finite precision. A simple calculation for an initial state of the work system as $\rho=(1-\epsilon)|W\rangle\langle W|+\epsilon| 0\rangle\langle 0|$ with Hamiltonian $H_{W}=$ $W|W\rangle\langle W|$ yields the bound

$$
\mathcal{V}_{\beta}\left(\rho, H_{W}\right) \leq-\log (\epsilon(1-\epsilon)) \quad \forall W .
$$

This implies that perfect cooling is impossible for any value of $W$ - even diverging - if the initial state of the work bit is a full-rank state.

\section{PROOF OF THEOREM 2}

We will now prove Theorem 2. Before we go into the details, let us first explain the general logic behind the result. It is clear that to obtain a single necessary and sufficient condition for cooling at low temperatures, we have to show that the infinite set of second laws in Eq. (10) collapses to a single condition. The first important step in the proof is the following lemma.

Lemma 5 (Concavity at low temperatures). Let $\beta>0$ and a Hamiltonian $H_{S}$ be given. There exists a critical inverse temperature $\beta_{\mathrm{cr}}$ such that for all $\beta_{S}>\beta_{\mathrm{cr}}$,

$$
\alpha \mapsto S_{\alpha}^{\prime \prime}\left(\omega_{\beta_{S}}\left(H_{S}\right) \| \omega_{\beta}\left(H_{S}\right)\right) \leq 0
$$

and

$$
S_{\infty}\left(\omega_{\beta_{S}}\left(H_{S}\right) \| \omega_{\beta}\left(H_{S}\right)\right) \leq \log Z_{\beta} .
$$

Here, the critical value $\delta\left(\beta_{S}\right)$ is given by

$$
\delta\left(\beta_{S}\right)=\frac{\log \left(Z_{\beta}\right)}{\mathcal{V}_{\beta}\left(\omega_{\beta_{S}}\left(H_{S}\right), H_{S}\right)} .
$$

Proof.-See Appendix A.

Using this result, we can now upper bound the Renyi divergence on the target by its linear approximation at the origin in this parameter regime. Since $S_{0}^{\prime}\left(\rho \| \omega_{\beta}(H)\right)=$ $S\left(\omega_{\beta}(H) \| \rho\right)=\mathcal{V}_{\beta}(\rho, H)$, we get

$$
S_{\alpha}\left(\omega_{\beta_{S}}\left(H_{S}\right) \| \omega_{\beta}\left(H_{S}\right)\right) \leq \mathcal{V}_{\beta}\left(\omega_{\beta_{S}}\left(H_{S}\right), H_{S}\right) \alpha, \quad \forall \alpha \leq \alpha_{c} .
$$

Second, for small enough target temperatures, we also have $S_{\infty}\left(\omega_{\beta_{S}}\left(H_{S}\right) \| \omega_{\beta}\left(H_{S}\right)\right) \leq \mathcal{V}_{\beta}\left(\omega_{\beta_{S}}\left(H_{S}\right), H_{S}\right) \alpha_{c}$. Since $\alpha \mapsto S_{\alpha}$ is monotonously increasing, the second laws in Eq. (10) are hence also satisfied if

$$
S_{\alpha}\left(\rho_{R} \| \omega_{\beta}\left(H_{R}\right)\right)>\mathcal{V}_{\beta}\left(\omega_{\beta_{S}}\left(H_{S}\right), H_{S}\right) \alpha, \quad \forall \alpha \leq \alpha_{c} .
$$

For small temperatures, we can further restrict the range of $\alpha$ to the interval $\left[0, \delta\left(\beta_{S}\right)\right)$, where $\delta\left(\beta_{S}\right)$ is given by

$$
\delta\left(\beta_{S}\right)=\frac{S_{\infty}\left(\omega_{\beta_{S}}\left(H_{S}\right) \| \omega_{\beta}\left(H_{S}\right)\right)}{\mathcal{V}_{\beta}\left(\omega_{\beta_{S}}\left(H_{S}\right), H_{S}\right)} .
$$

The final step is now given by bounding the Renyi divergence of the resource $S_{\alpha}\left(\rho_{R} \| \omega_{\beta}\left(H_{R}\right)\right)$. In particular, if we knew that it was convex (such as in the case of a thermal resource with $E_{\beta}^{R}$ being convex), we could lower bound it by its linear approximation at the origin and obtain the necessary and sufficient condition (11).

In the general case, $S_{\alpha}\left(\rho_{R} \| \omega_{\beta}\left(H_{R}\right)\right)$ will not be convex. But we only have to check small values of $\alpha<\delta\left(\beta_{S}\right)$ and simply Taylor expand $S_{\alpha}\left(\rho_{R} \| \omega_{\beta}\left(H_{R}\right)\right)$. Using Taylor's theorem, we then obtain

$S_{\alpha}\left(\rho_{R} \| \omega_{\beta}\left(H_{R}\right)\right) \geq \mathcal{V}_{\beta}\left(\rho_{R}, H_{R}\right) \alpha-k\left(\beta_{S}, \beta, \rho_{R}, H_{R}\right) \alpha^{2}$.

This yields as a new sufficient condition

$$
\mathcal{V}_{\beta}\left(\rho_{R}, H_{R}\right) \alpha-k\left(\beta_{S}, \beta, \rho_{R}, H_{R}\right) \alpha^{2} \geq \mathcal{V}_{\beta}\left(\omega_{\beta_{S}}\left(H_{S}\right), H_{S}\right) \alpha,
$$

for all $0<\alpha \leq \delta\left(\beta_{S}\right)$. The function $k\left(\beta_{S}, \beta, \rho_{R}, H_{R}\right) \geq 0$ is given by

$$
k\left(\beta_{S}, \beta, \rho_{R}, H_{R}\right)=\max \left\{0,-\min _{\alpha \leq \delta\left(\beta_{S}\right)} S_{\alpha}^{\prime \prime}\left(\rho_{R} \| \omega_{\beta}\left(H_{R}\right)\right)\right\} .
$$

We can now divide the sufficient condition by $\alpha$ and, since $k\left(\beta_{S}, \beta, \rho_{R}, H_{R}\right) \geq 0$, replace $\alpha$ by $\delta\left(\beta_{S}\right)$ to arrive at the final sufficient condition

$$
\mathcal{V}_{\beta}\left(\rho_{R}, H_{R}\right)-K\left(\beta_{S}, \beta, \rho_{R}, H_{R}, H_{S}\right) \geq \mathcal{V}_{\beta}\left(\omega_{\beta_{S}}\left(H_{S}\right), H_{S}\right),
$$

with $K\left(\beta_{S}, \beta, \rho_{R}, H_{R}, H_{S}\right)=k\left(\beta_{S}, \beta, \rho_{R}, H_{R}\right) \delta\left(\beta_{S}\right)$. This finishes the proof.

\section{SUMMARY}

In this work, we have investigated the limits on lowtemperature cooling when arbitrary systems out of equilibrium are used as a resource. We provide sufficient and necessary conditions that establish novel upper and lower bounds on the amount of resources that are needed to cool a system close to its ground state. We found that the limitations are ruled by a single quantity, namely, the vacancy. This is remarkable since at higher temperatures, there is an infinite family of "second laws" that need to be checked to determine whether a nonequilibrium state transition is possible.

We have only focused on the amount of nonequilibrium resources, as we assume access to an infinite heat bath, and we leave considerations about the time and complexity of the cooling protocol aside. These other kinds of resources have been explored in other complementary works on the third law [3-6]. It would be interesting to see if the vacancy 
plays a role to express the limitations on the size of the heat bath or any other resources that diverge when cooling a system to absolute zero. More particularly, it is an interesting question for future work to obtain the optimal sufficient scaling of the size of the heat bath and the potential "catalyst" $\tau$ that is needed to cool the system to the final low temperature [54]. In this work, we have required the catalyst to be returned exactly. The necessary condition (11) and the resulting quantitative unattainability principle are, however, stable when one instead requires that the vacancy of the catalyst only changes a little (see Appendix $G$ for a discussion of approximate catalysts). We leave studying how the sufficient condition behaves in such an approximate scenario to future work.

The results of Sec. V suggest that for a large class of physically relevant systems, the third law can be expressed simply as the monotonicity of the vacancy. It would be of interest to specify more general assumptions on a manybody system so that this is the case. On the other hand, there exist systems for which the vacancy is not a sufficient condition. This opens the possibility to have families of resources that, although out of equilibrium, are useless for cooling. We leave this as an open question for future work.

Lastly, we note that, in this work, we have focused on the expenditure of nonequilibrium resources for lowtemperature cooling, which are precisely the resources that are employed in laser cooling [55]. An interesting open research direction is to analyze protocols of laser cooling in the light of the bounds obtained here.

\section{ACKNOWLEDGMENTS}

We thank Lluis Masanes, Joseph M. Renes, and Jens Eisert for interesting discussions and valuable feedback. This work has been supported by the European Research Council (ERC) (TAQ), the Deutsche Forschungsgemeinschaft (DFG) (GA 2184/2-1), and the Studienstiftung des Deutschen Volkes.

\section{APPENDIX A: PROOF OF CONCAVITY OF RENYI DIVERGENCE FOR LOW TEMPERATURES}

In this section, we prove Lemma 5, which is about the concavity of the Renyi divergence at low temperatures. The lemma holds for any Hamiltonian with a pure point spectrum, a gap above the ground state, and the property that the partition sum exists for any positive temperature.

Lemma 6 (Concavity at low temperatures). Let $\beta>0$ and a Hamiltonian $H_{S}$ with ground-state degeneracy $g_{0}$ be given. There exists a critical inverse temperature $\beta_{\mathrm{cr}}$ such that, for all $\beta_{S}>\beta_{\text {cr }}$ and for all $0<\alpha<\delta\left(\beta_{S}\right)$, we have

$$
\alpha \mapsto S_{\alpha}^{\prime \prime}\left(\omega_{\beta_{S}} \| \omega_{\beta}\right) \leq 0
$$

and

$$
S_{\infty}\left(\omega_{\beta_{S}} \| \omega_{\beta}\right) \leq \log Z_{\beta}
$$

Here, the critical value $\delta\left(\beta_{S}\right)$ is given by

$$
\delta\left(\beta_{S}\right)=\frac{\log \left(Z_{\beta}\right)}{\mathcal{V}_{\beta}\left(\omega_{\beta_{S}}\left(H_{S}\right), H_{S}\right)}<1 .
$$

Proof-Let us first prove that the max-Renyi divergence is upper bounded by the partition function at the bath's temperature $\beta$. Suppose that $\beta_{S}>\beta$, and let us write

$$
\begin{aligned}
S_{\alpha}\left(\omega_{\beta_{S}}\left(H_{S}\right) \| \omega_{\beta}\left(H_{S}\right)\right) \\
=\frac{1}{\alpha-1} \log \left(\sum_{i} g_{i} \mathrm{e}^{-\alpha\left(\beta_{S}-\beta\right) E_{i}}\left(Z_{\beta} / Z_{\beta_{S}}\right)^{\alpha} \frac{\mathrm{e}^{-\beta E_{i}}}{Z_{\beta}}\right) \\
=\frac{1}{\alpha-1} \log \left(\mathrm{e}^{-\alpha\left(\beta_{S}-\beta\right) E_{0}}\left(Z_{\beta} / Z_{\beta_{S}}\right)^{\alpha}\right. \\
\left.\quad \times \sum_{i} g_{i} \mathrm{e}^{-\alpha\left(\beta_{S}-\beta\right)\left(E_{i}-E_{0}\right)} \frac{\mathrm{e}^{-\beta E_{i}}}{Z_{\beta}}\right),
\end{aligned}
$$

where $E_{i}$ denotes the different energies of $H_{S}$, with degeneracies $g_{i}$. Assuming without loss of generality $E_{0}=0$, we write this as

$$
\begin{aligned}
S_{\alpha}\left(\omega_{\beta_{S}}\left(H_{S}\right) \| \omega_{\beta}\left(H_{S}\right)\right) \\
=\frac{\alpha}{\alpha-1} \log \left(Z_{\beta} / Z_{\beta_{S}}\right) \\
\quad+\frac{1}{\alpha-1} \log \left(1+\sum_{i>0} \mathrm{e}^{-\alpha\left(\beta_{S}-\beta\right) E_{i}} \frac{\mathrm{e}^{-\beta E_{i}}}{Z_{\beta}}\right) .
\end{aligned}
$$

It is now obvious that, in the limit, we obtain

$$
\begin{aligned}
S_{\infty}\left(\omega_{\beta_{S}}\left(H_{S}\right) \| \omega_{\beta}\left(H_{S}\right)\right) & =\lim _{\alpha \rightarrow \infty} S_{\alpha}\left(\omega_{\beta_{S}}\left(H_{S}\right) \| \omega_{\beta}\left(H_{S}\right)\right) \\
& =\log \left(Z_{\beta}\right)-\log \left(Z_{\beta_{S}}\right) \leq \log Z_{\beta} .
\end{aligned}
$$

As a second step, let us find the condition for which $\delta\left(\beta_{S}\right)<1$. We express the vacancy as

$$
\mathcal{V}_{\beta}\left(\omega_{\beta_{S}}\left(H_{S}\right), H_{S}\right)=\beta_{S} E_{\beta}-S_{\beta}+\log Z_{\beta_{S}},
$$

where we write $S_{\beta}:=S\left(\omega_{\beta}\left(H_{S}\right)\right)$. We thus need

$$
\beta_{S} E_{\beta}-S\left(\omega_{\beta}\left(H_{S}\right)\right)>\log Z_{\beta}-\log Z_{\beta_{S}} .
$$

Relaxing to the sufficient criterion $\beta_{S} E_{\beta}-S_{\beta}>\log Z_{\beta}=$ $S_{\beta}-\beta E_{\beta}$, we thus obtain

$$
\beta_{S}>\frac{2 S_{\beta}-\beta E_{\beta}}{E_{\beta}} .
$$

Let us now turn to the concavity. We use the representation of $S_{\alpha}^{\prime \prime}$ proven in the next section, which is given by 


$$
\begin{aligned}
S_{\alpha}^{\prime \prime}\left(\omega_{\beta_{S}} \| \omega_{\beta}\right)= & \frac{2}{(1-\alpha)^{3}}\left(\log Z_{\beta_{S}}-\log Z_{\tilde{\beta}(\alpha)}\right. \\
& +\left(\beta_{S}-\tilde{\beta}(\alpha)\right) E_{\tilde{\beta}(\alpha)} \\
& \left.-\left(\beta_{S}-\tilde{\beta}(\alpha)\right)^{2} \operatorname{Var}(H)_{\tilde{\beta}(\alpha)}\right),
\end{aligned}
$$

where $\tilde{\beta}(\alpha)=\beta(1-\alpha)+\alpha \beta_{S}$. Since we are only interested in $\alpha<\delta\left(\beta_{S}\right)<1$, we have $\beta \leq \tilde{\beta}(\alpha)<\beta_{S}$. We therefore have to show that the terms in the parentheses are negative. Let us use the fact that the average energy is monotonic with $\beta$ and that $Z_{\tilde{\beta}(\alpha)}>1$ to bound these terms as

$$
\begin{aligned}
\frac{(1-\alpha)^{3}}{2} S_{\alpha}^{\prime \prime}\left(\omega_{\beta_{S}} \| \omega_{\beta}\right) & \leq \log Z_{\beta_{S}}+\left(\beta_{S}-\tilde{\beta}(\alpha)\right) E_{\tilde{\beta}(\alpha)}-\left(\beta_{S}-\tilde{\beta}(\alpha)\right)^{2} \operatorname{Var}(H)_{\tilde{\beta}(\alpha)} \\
& \leq \log Z_{\beta_{S}}+\left(\beta_{S}-\beta\right) E_{\beta}-\left(\beta_{S}-\tilde{\beta}(\alpha)\right)^{2} \operatorname{Var}(H)_{\tilde{\beta}(\alpha)} \\
& \leq \log (d)+\left(\beta_{S}-\beta\right) E_{\max }-\left(\beta_{S}-\tilde{\beta}(\alpha)\right)^{2} \min _{x \in[\beta, \tilde{\beta}(\alpha)]} \operatorname{Var}(H)_{x} .
\end{aligned}
$$

Now, we bound $\tilde{\beta}(\alpha)$ by using $\tilde{\beta}(\alpha) \leq \tilde{\beta}\left(\delta\left(\beta_{S}\right)\right)=$ : $\tilde{\beta}^{*}\left(\beta_{S}\right)$. It is clear that if we can bound $\tilde{\beta}^{*}\left(\beta_{S}\right)$ by a constant, the terms in the parentheses become negative for some $\beta_{S}$ since the second-order term in $\beta_{S}$ dominates. To see that $\tilde{\beta}^{*}\left(\beta_{S}\right)$ is indeed upper bounded by a constant, we again write the vacancy as

$$
\mathcal{V}_{\beta}\left(\omega_{\beta_{S}}\left(H_{S}\right), H_{S}\right)=-S\left(\omega_{\beta}\right)+\beta_{S} E_{\beta}+\log Z_{\beta_{S}}
$$

to obtain

$$
\begin{aligned}
\beta^{*} & :=\lim _{\beta_{S} \rightarrow \infty} \tilde{\beta}^{*}\left(\beta_{S}\right) \\
& =\lim _{\beta_{S} \rightarrow \infty} \beta\left(1-\delta\left(\beta_{S}\right)\right)+\delta\left(\beta_{S}\right) \beta_{S} \quad(\mathrm{~A} 14) \\
& =\beta+\lim _{\beta_{S} \rightarrow \infty} \frac{\log Z_{\beta}}{\beta_{S} E_{\beta}+\log Z_{\beta_{S}}-S\left(\omega_{\beta}\left(H_{S}\right)\right)} \beta_{S} \\
& =\beta+\frac{\log Z_{\beta}}{E_{\beta}} .
\end{aligned}
$$

This finishes the proof that $\beta_{\mathrm{cr}}$ exists as claimed in the lemma. We also note that the function $\tilde{\beta}^{*}\left(\beta_{S}\right)$ is monotonically decreasing for all $\beta_{S}$ such that $\tilde{\beta}^{*}\left(\beta_{S}\right)<1$. Finally, note that Eq. (A12) allows us to give upper bounds on $\beta_{\text {cr }}$ once we have lower bounds on the energy variance for inverse temperatures in the interval $\left[\beta, \beta^{*}\right]$.

\section{APPENDIX B: RENYI DIVERGENCE BETWEEN THERMAL STATES}

Here, we specialize to the situation where the resource states are thermal, with inverse temperature $\beta_{R}$. We now calculate the Renyi divergence for $\alpha<1$ in this case. We first write

$$
\begin{gathered}
S_{\alpha}\left(\omega_{\beta_{R}} \| \omega_{\beta}\right)=-\frac{\alpha}{\alpha-1} \log Z_{\beta_{R}}+\log Z_{\beta} \\
+\frac{1}{\alpha-1} \log \operatorname{Tr}\left(\mathrm{e}^{-\beta_{R} H \alpha} \mathrm{e}^{-\beta H(1-\alpha)}\right) \\
=-\frac{\alpha}{\alpha-1} \log Z_{\beta_{R}}+\log Z_{\beta}+\frac{1}{\alpha-1} \log Z_{\left(\beta_{R}-\beta\right) \alpha+\beta} \\
=-\frac{\alpha-1}{\alpha-1} \log Z_{\beta_{R}}+\log Z_{\beta}+\frac{1}{\alpha-1} \log \left(Z_{\left(\beta_{R}-\beta\right) \alpha+\beta} / Z_{\beta_{R}}\right) \\
=\log \left(Z_{\beta} / Z_{\beta_{R}}\right)+\frac{1}{\alpha-1} \log \left(Z_{\left(\beta_{R}-\beta\right) \alpha+\beta} / Z_{\beta_{R}}\right) .
\end{gathered}
$$

We now show that the function is convex, provided $\beta_{R}<\beta$ and that the function $x \mapsto E_{\beta_{R}+x}$ is convex for $0 \leq x \leq \beta-\beta_{R}$. For the second derivative [with $\tilde{\beta}=$ $\left.\left(\beta_{R}-\beta\right) \alpha+\beta\right]$, we obtain

$$
\begin{aligned}
S_{\alpha}\left(\omega_{\beta_{R}} \| \omega_{\beta}\right)^{\prime \prime} & =\frac{2}{(1-\alpha)^{3}} \log Z_{\beta_{R}}-\frac{2}{(1-\alpha)^{3}} \log Z_{\tilde{\beta}}-2 \frac{1}{(1-\alpha)^{2}} \partial_{\alpha} \log Z_{\tilde{\beta}}+\frac{1}{\alpha-1} \partial_{\alpha}^{2} \log Z_{\tilde{\beta}} \\
& =\frac{2}{(1-\alpha)^{3}} \log Z_{\beta_{R}}-\frac{2}{(1-\alpha)^{3}} \log Z_{\tilde{\beta}}-2 \frac{1}{(1-\alpha)^{2}}\left(\beta-\beta_{R}\right) E_{\tilde{\beta}}-\frac{1}{1-\alpha}\left(\beta-\beta_{R}\right)^{2} \operatorname{Var}(H)_{\tilde{\beta}} \\
& =\frac{2}{(1-\alpha)^{3}}\left[\log Z_{\beta_{R}}-\log Z_{\tilde{\beta}}-(1-\alpha)\left(\beta-\beta_{R}\right) E_{\tilde{\beta}}-\frac{(1-\alpha)^{2}}{2}\left(\beta-\beta_{R}\right)^{2} \operatorname{Var}(H)_{\tilde{\beta}}\right] .
\end{aligned}
$$


Utilizing $(1-\alpha)\left(\beta-\beta_{R}\right)=\tilde{\beta}-\beta_{R}$, we can write this as

$$
\begin{aligned}
S_{\alpha}\left(\omega_{\beta_{R}} \| \omega_{\beta}\right)^{\prime \prime}= & \frac{2}{(1-\alpha)^{3}}\left[\log Z_{\beta_{R}}-\log Z_{\tilde{\beta}}-\left(\tilde{\beta}-\beta_{R}\right) E_{\tilde{\beta}}\right. \\
& \left.-\frac{\left(\tilde{\beta}-\beta_{R}\right)^{2}}{2} \operatorname{Var}(H)_{\tilde{\beta}}\right] .
\end{aligned}
$$

Here, we have introduced the average energy $E_{\beta}$ and the variance $\operatorname{Var}(H)_{\beta}=\left\langle H^{2}\right\rangle_{\beta}-\langle H\rangle_{\beta}^{2}$, which fulfill $\partial_{x} E_{x}=$ $-\operatorname{Var}(H)_{x}$. With these expressions at hand, we now show Theorem 3 and another result about the convexity of Renyi divergences for sufficiently large reference temperature $\beta$.

\section{Proof of Theorem 3}

We need to show that the right-hand side of Eq. (B8) is positive with the premise that $x \mapsto E_{x}$ is convex in $x \in\left[\beta_{R}, \beta\right], \beta_{R} \leq \beta$, and $\alpha<1$. This last condition on $\alpha$ implies that we need to show that

$\log Z_{\beta_{R}}-\log Z_{\tilde{\beta}} \geq\left(\tilde{\beta}-\beta_{R}\right) E_{\tilde{\beta}}+\frac{\left(\tilde{\beta}-\beta_{R}\right)^{2}}{2} \operatorname{Var}(H)_{\tilde{\beta}}$.

We use an integral representation on the left-hand side:

$$
\begin{aligned}
\log Z_{\beta_{R}}-\log Z_{\tilde{\beta}} & =-\int_{0}^{\tilde{\beta}-\beta_{R}} \frac{\mathrm{d}}{\mathrm{d} x} \log Z_{\beta_{R}+x} \mathrm{~d} x \\
& =\int_{0}^{\tilde{\beta}-\beta_{R}} E_{\beta_{R}+x} \mathrm{~d} x .
\end{aligned}
$$

Hence, we conclude that

$$
\int_{0}^{\tilde{\beta}-\beta_{R}} E_{\beta_{R}+x} \mathrm{~d} x \geq\left(\tilde{\beta}-\beta_{R}\right) E_{\tilde{\beta}}+\frac{\left(\tilde{\beta}-\beta_{R}\right)^{2}}{2} \operatorname{Var}(H)_{\tilde{\beta}} .
$$

Whether this inequality is satisfied, and thus, $S_{\alpha}\left(\rho_{R} \| \omega_{\beta}(H)\right)$ is convex, is entirely determined by the function $x \mapsto E_{x}$. This is due to the fact that the derivative of $E_{x}$ is given by $-\operatorname{Var}(H)_{x}$, so the right-hand side can be seen as a linear approximation to the function $E_{x}$. A geometrical interpretation is provided in Fig. 2, showing that it is trivially satisfied when $E_{x}$ is convex. This finishes the proof.

As a final remark, although not useful to obtain bounds on the third law, we note that a completely analogous argument implies that if $\alpha<1, E_{x}$ is convex, but in contrast to the previous case, $\beta_{R} \geq \beta$, then we get

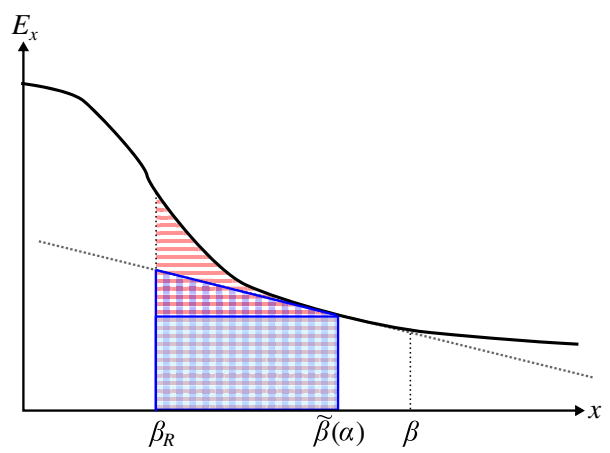

FIG. 2. The left-hand side of Eq. (B11) is represented by the red striped area under the curve. The right-hand side corresponds to the blue striped region. This can be seen by noting that the blue striped region can be decomposed into a rectangle of sides $\tilde{\beta}-\beta_{R}$ and $E_{\tilde{\beta}}$ (light blue), which corresponds to the first term on the right-hand side of Eq. (B11), and a triangle that corresponds to the second term. If the function is $E_{x}$ convex, the red region is always larger than the blue region.

$$
\int_{0}^{\tilde{\beta}-\beta_{R}} E_{\beta_{R}+x} \mathrm{~d} x \leq\left(\tilde{\beta}-\beta_{R}\right) E_{\tilde{\beta}}+\frac{\left(\tilde{\beta}-\beta_{R}\right)^{2}}{2} \operatorname{Var}(H)_{\tilde{\beta}}
$$

This shows that, in the case of resources colder than the bath, the function $S_{\alpha}\left(\rho_{R} \| \omega_{\beta}(H)\right)$ is concave.

\section{Very cold heat baths}

We now show that in the case of very cold heat baths (very large $\beta$ ), we also have that $S_{\alpha}\left(\omega_{\beta_{R}} \| \omega_{\beta}\right)$ is convex, and hence, Eq. (11) becomes sufficient and necessary.

Theorem 7. For any resource of the form $\left(\omega_{\beta_{R}}\left(H_{R}\right), H_{R}\right)$, given a fixed $\beta_{R}$, there exists a sufficiently large value of $\beta$ such that Eq. (11) is a sufficient and necessary condition for low-temperature cooling.

Proof-We only give a sketch and show that $S_{\alpha}\left(\omega_{\beta_{R}} \| \omega_{\beta}\right)$ is convex for values of $\alpha<\alpha_{c}$, where $\alpha_{c}<1$ is chosen arbitrarily. Recalling Eq. (B11), we then need to show that

$$
\int_{0}^{\tilde{\beta}-\beta_{R}} E_{\beta_{R}+x} \mathrm{~d} x \geq\left(\tilde{\beta}-\beta_{R}\right) E_{\tilde{\beta}}+\frac{\left(\tilde{\beta}-\beta_{R}\right)^{2}}{2} \operatorname{Var}(H)_{\tilde{\beta}}
$$

Note that in the limit of large $\beta$, the scaling of the right-hand side of Eq. (B13) is such that $\tilde{\beta}-\beta_{R}=(1-\alpha)\left(\beta-\beta_{R}\right)$ scales proportionally to $\beta$, while $E_{\tilde{\beta}}$ and $\operatorname{Var}(H)_{\tilde{\beta}}$ scale as $e^{-k \beta}$. Therefore, the right-hand side of Eq. (B13) approaches zero as $\beta \rightarrow \infty$, whereas the left-hand side grows monotonically with $\beta$. Hence, Eq. (B13) is fulfilled, which concludes the proof. 


\section{APPENDIX C: EQUIDISTANT LEVELS}

Here, we consider the particular case of a system with $M+1$ equidistant levels and show that the function $E_{\beta}$ is convex. The energy gap between subsequent levels is $\Delta$, and we set the ground-state energy to zero. The energy $E_{\beta}$ then takes the form

$$
E_{\beta}=\frac{1}{\mathrm{e}^{\beta \Delta}-1} \Delta-\frac{M+1}{\mathrm{e}^{(M+1) \Delta \beta}-1} \Delta .
$$

In particular, for $M \rightarrow \infty$, we obtain results for the harmonic oscillator, and for $M=1$, we obtain results for a qubit. We have to prove that the second derivative is positive, i.e.,

$$
\begin{aligned}
E_{\beta}^{\prime \prime}= & \frac{1}{8} \Delta^{3}\left[\frac{\sinh (\beta \Delta)}{\sinh (\beta \Delta / 2)^{4}}\right. \\
& -\underbrace{(M+1)^{3} \frac{\sinh ((M+1) \beta \Delta)}{\sinh ((M+1) \beta \Delta / 2)^{4}}}_{=: f(\beta, M+1)}] \geq 0 .
\end{aligned}
$$

For $M=0$, this is clearly true. We set $M+1=: \gamma$ and show that $\partial_{\gamma} f(\beta, \gamma) \leq 0$. We have

$$
\begin{aligned}
\partial_{\gamma} f(\beta, \gamma)= & -\gamma^{2} \frac{1}{\sinh (\gamma \beta \Delta / 2)^{4}}[\gamma \beta \Delta(2+\cosh (\gamma \beta \Delta)) \\
& -3 \sinh (\gamma \beta \Delta)] .
\end{aligned}
$$

In the following, set $\gamma \beta \Delta=x$. Because of the negative prefactor, we are done if we can show

$$
x(2+\cosh (x))-3 \sinh (x) \geq 0 .
$$

We show this using a Taylor expansion:

$$
\begin{aligned}
2 x & +x \cosh (x)-3 \sinh (x) \\
& =2 x+\sum_{n=0}^{\infty} x^{2 n+1}\left(\frac{1}{(2 n) !}-\frac{3}{(2 n+1) !}\right) \\
& =2 x+\sum_{n=0}^{\infty} x^{2 n+1}\left(\frac{((2 n+1)-3)(2 n) !}{(2 n) !(2 n+1) !}\right) \\
& =2 x-2 x+\sum_{n=1}^{\infty} x^{2 n+1}\left(\frac{((2 n+1)-3)(2 n) !}{(2 n) !(2 n+1) !}\right)
\end{aligned}
$$

$\geq 0$.

\section{APPENDIX D: WORK-SOURCE MODEL}

Here, we show that a work source of the form $\left(\rho_{R}^{w}, H_{R}^{w}\right)$ as given in the main text fulfills the second law of thermodynamics. Let us consider an arbitrary system $\left(\rho_{S}, H_{S}\right)$, and let us consider catalytic thermal operations on $S W$. We show that the maximum amount of mean energy that one can store on the work source is bounded by the initial nonequilibrium free energy of $S$. Let us recall (see, for instance, Ref. [16]) that the free-energy difference is given by

$\Delta F_{\beta}(\rho, H):=1 / \beta S\left(\rho \| \omega_{\beta}(H)\right)=F(\rho, H)-F\left(\omega_{\beta}(H)\right)$,

where $F(\rho, H)=\operatorname{Tr}(\rho H)-(1 / \beta) S(\rho)$ is the free energy. form

The protocol of work extraction is a transition of the

$$
\rho_{S W}^{i}:=\rho_{S} \otimes \rho_{R}^{w} \rightarrow \rho_{S W}^{f}=\mathcal{E}\left(\rho_{S W}^{i}\right),
$$

where $\mathcal{E}$ is any channel that has the Gibbs state as a fixed point. Monotonicity of $\Delta F_{\beta}$ under channels of the form $\mathcal{E}$ implies that

$$
\begin{aligned}
\Delta F_{\beta}^{f} & :=\Delta F_{\beta}\left(\rho_{S} R^{f}, H_{S}+H_{R}\right) \leq \Delta F_{\beta}\left(\rho_{S R}^{i}, H_{S}+H_{R}\right) \\
& :=\Delta F_{\beta}^{i} .
\end{aligned}
$$

Combining this last equation with superadditivity and additivity of the relative entropy, one can easily find, following a similar reasoning as in Ref. [16], that

$$
\Delta E_{R} \leq \Delta F_{\beta}\left(\rho_{S}, H_{S}\right),
$$

where $\quad \Delta E_{R}=\operatorname{Tr}\left(\llbracket \otimes H_{R}^{w} \rho_{S R}^{f}\right)-\operatorname{Tr}\left(\llbracket \otimes H_{R}^{w} \rho_{S R}^{i}\right) \quad$ is $\quad$ the mean energy stored in the work source $R$.

\section{APPENDIX E: ARBITRARY TARGET STATES CLOSE TO THE GROUND STATE}

In this section, we prove a result similar to our general sufficient condition for cooling, but we consider target states of the form

$$
\rho_{\epsilon}=(1-\epsilon)|0\rangle\langle 0|+\epsilon \rho^{\perp}, \quad \epsilon \ll 1,
$$

where $\rho^{\perp}$ is a density matrix that has full rank on the subspace orthogonal to the ground state $|0\rangle$ and commutes with the Hamiltonian $H_{S}$.

Theorem 8 (General sufficient condition for cooling). For every choice of $\beta, H_{S}$, and $\rho^{\perp}$ as above, there is a critical $\epsilon_{\mathrm{cr}}>0$ such that for any $\epsilon<\epsilon_{\mathrm{cr}}$ the condition

$\mathcal{V}_{\beta}\left(\rho_{R}, H_{R}\right)+\tilde{K}\left(\epsilon, \beta, \rho_{R}, H_{R}, H_{S}, \rho^{\perp}\right) \geq \mathcal{V}_{\beta}\left(\rho_{\epsilon}, H_{S}\right)$ 
is sufficient for cooling. The function $\tilde{K}$ has the property $\tilde{K}\left(\epsilon, \beta, \rho_{R}, H_{R}, \rho^{\perp}\right) \rightarrow 0$ as $\epsilon \rightarrow \infty$ for any fixed $\beta, H_{R}, \rho_{R}$, $\rho^{\perp}$, and $H_{S}$.

The proof of this theorem is essentially identical to the one of Theorem 2. The only difference is that instead of Lemma 5, we use the following lemma:

Lemma 9 (Concavity close to ground state). Let $H_{S}$ be a $d$-dimensional Hamiltonian with ground state $|0\rangle$ and $H|0\rangle=0$. Let $\beta>0$ be fixed, and consider the state

$$
\rho_{\epsilon}=(1-\epsilon)|0\rangle\langle 0|+\epsilon \rho^{\perp},
$$

with $\operatorname{rank}\left(\rho^{\perp}\right)=d-1, \rho^{\perp}|0\rangle=0$ and $\left[\rho^{\perp}, H_{S}\right]=0$. Then, there exists an $\epsilon_{\mathrm{cr}}>0$ such that for all $\alpha<\delta(\epsilon)$,

$$
\frac{\mathrm{d}^{2}}{\mathrm{~d} \alpha^{2}} S_{\alpha}\left(\rho_{\epsilon} \| \omega_{\beta}\left(H_{S}\right)\right)<0, \quad \forall \epsilon<\epsilon_{\mathrm{cr}}
$$

Here, $\delta(\epsilon)$ fulfills

$$
\delta(\epsilon)=\frac{\log Z_{\beta}}{\mathcal{V}_{\beta}\left(\rho_{\epsilon}, H_{S}\right)}<1, \quad \forall \epsilon<\epsilon_{\mathrm{cr}} .
$$

We now prove this lemma. Let us express the Renyi divergence as

$$
\begin{aligned}
S_{\alpha}\left(\rho_{\epsilon} \| \omega_{\beta}\left(H_{S}\right)\right)= & \frac{1}{\alpha-1} \log \left((1-\epsilon)^{\alpha}\right. \\
& \left.+\epsilon^{\alpha} \operatorname{Tr}\left(\left(\rho^{\perp}\right)^{\alpha} \mathrm{e}^{-\beta H_{S}(1-\alpha)}\right)\right)+\log \left(Z_{S}\right) \\
= & \frac{1}{\alpha-1} \log (f(\alpha))+\log \left(Z_{S}\right)
\end{aligned}
$$

As is apparent from the expression, in the following, we often encounter the functions

$$
\begin{gathered}
\tilde{f}(\alpha):=\operatorname{Tr}\left(\left(\rho^{\perp}\right)^{\alpha} \mathrm{e}^{-\beta H(1-\alpha)}\right), \\
f_{\epsilon}(\alpha):=\operatorname{Tr}\left(\rho_{\epsilon}^{\alpha} \mathrm{e}^{-\beta H(1-\alpha)}\right)=(1-\epsilon)^{\alpha}+\epsilon^{\alpha} \tilde{f}(\alpha) .
\end{gathered}
$$

It is useful to remember from the main text that $\rho^{\perp}$ is a normalized quantum state that commutes with $H$ and has rank $d-1$. In the following, we also often write $S_{\alpha}$ instead of $S_{\alpha}\left(\rho_{\epsilon} \| \omega_{\beta}\left(H_{S}\right)\right)$ and simply $f_{\epsilon}$ or $f_{\epsilon, \alpha}$ instead of $f_{\epsilon}(\alpha)$ to simplify the notation (similarly for $\tilde{f}$ ). While $f_{\epsilon}$ and $\tilde{f}$ are structurally essentially the same, it is important to keep in mind that only $f_{\epsilon}$, and not $\tilde{f}$, depends on $\epsilon$.

We now have to prove that $S_{\alpha}$ is concave for small enough $\epsilon$; i.e., we have to show that there exists a $\epsilon_{\mathrm{cr}}>0$ such that its second derivative is negative for $\epsilon<\epsilon_{\mathrm{cr}}$. The second derivative of $S_{\alpha}$ can be computed straightforwardly, and it gives
$S_{\alpha}^{\prime \prime}=-\frac{2}{(1-\alpha)^{3}} \log f_{\epsilon}-\frac{2}{(1-\alpha)^{2}} \frac{f_{\epsilon}^{\prime}}{f_{\epsilon}}+\frac{1}{1-\alpha}\left(\left(\frac{f_{\epsilon}^{\prime}}{f_{\epsilon}}\right)^{2}-\frac{f_{\epsilon}^{\prime \prime}}{f_{\epsilon}}\right)$.

To proceed, we need to establish a few properties of functions like $f_{\epsilon}$ and $\tilde{f}$. We collect these properties in a series of lemmata.

Lemma 10. Let $\rho$ be a quantum state and $\sigma$ be a positive semidefinite operator with $[\rho, \sigma]=0$. Define $f(\alpha):=\operatorname{Tr}\left(\rho^{\alpha} \sigma^{1-\alpha}\right)$. Then,

$$
\left(f^{\prime}\right)^{2}-f^{\prime \prime} f \leq 0, \quad 0<\alpha<1 .
$$

Proof.-A simple calculation shows that

$$
f^{\prime}(\alpha)=\operatorname{Tr}\left[\rho^{\alpha}(\log (\rho)-\log (\sigma)) \sigma^{1-\alpha}\right],
$$

$$
f^{\prime \prime}(\alpha)=\operatorname{Tr}\left[\rho^{\alpha}(\log (\rho)-\log (\sigma))^{2} \sigma^{1-\alpha}\right] .
$$

We now use the Cauchy-Schwarz inequality $\left|\operatorname{Tr}\left(A^{\dagger} B \rho\right)\right|^{2} \leq$ $\operatorname{Tr}\left(A^{\dagger} A \rho\right) \operatorname{Tr}\left(B^{\dagger} B \rho\right)$ with $A=\rho^{-\alpha / 2}(\log (\rho)-\log (\sigma)) \sigma^{\alpha / 2}$ and $B=\rho^{-\alpha / 2} \sigma^{\alpha / 2}$ to obtain (note the change from $\alpha$ to $1-\alpha$ )

$$
\begin{aligned}
f^{\prime}(1-\alpha)^{2} & =\operatorname{Tr}\left[\rho^{-\alpha / 2}(\log (\rho)-\log (\sigma)) \sigma^{\alpha / 2} \rho^{-\alpha / 2} \sigma^{\alpha / 2} \rho\right]^{2} \\
& \leq \operatorname{Tr}\left[\rho^{-\alpha}(\log (\rho)-\log (\sigma))^{2} \sigma^{\alpha} \rho\right] \operatorname{Tr}\left(\rho^{-\alpha} \sigma^{\alpha} \rho\right) \\
& =\operatorname{Tr}\left[\rho^{1-\alpha}(\log (\rho)-\log (\sigma))^{2} \sigma^{\alpha}\right] \operatorname{Tr}\left(\rho^{1-\alpha} \sigma^{\alpha}\right) \\
& =f^{\prime \prime}(1-\alpha) f(1-\alpha) .
\end{aligned}
$$

Lemma 11. Let $H$ be a Hamiltonian with ground-state energy $E_{0}=0$ and let $\sigma$ be a quantum state with $[\sigma, H]=0$. Then,

$$
f(\alpha):=\operatorname{Tr}\left(\sigma^{\alpha} \mathrm{e}^{-\beta H(1-\alpha)}\right) \leq Z, \quad 0 \leq \alpha \leq 1 .
$$

Proof.-From the calculation of the previous lemma, we see that the second derivative of $f$ is the trace of a product of positive commuting operators. Hence, it is always positive, and therefore $f$ is convex. But since $H \geq 0$, we have $f(0)=Z \geq 1=f(1)$, and from convexity, we get $f(\alpha) \leq Z$.

Note that because of our assumption about the ground-state energy, we have $Z_{S} \geq 1$, and from the above lemma, we know $f_{\epsilon} \leq Z_{S}$. We now show that for every $0<\alpha_{c}^{\prime}<1$, we have $1 \leq f_{\epsilon}(\alpha) \leq Z_{S}$ if $\epsilon$ is small enough and $\alpha<\alpha_{c}^{\prime}$. 
Lemma 12. For any $0<\alpha_{c}^{\prime}<1$, there exists a critical $\epsilon_{\mathrm{cr}}^{\prime}>0$, such that for all $\epsilon<\epsilon_{\mathrm{cr}}^{\prime}$, we have

$$
f_{\epsilon}(\alpha):=\operatorname{Tr}\left(\rho_{\epsilon}^{\alpha} \mathrm{e}^{-\beta H(1-\alpha)}\right) \geq 1, \quad 0 \leq \alpha<\alpha_{c}^{\prime} .
$$

Proof.-Assume some $0<\alpha<\alpha_{c}^{\prime}$. Using the fact that $\tilde{f}$ is independent of $\epsilon$ and positive, we can lower bound it by some $\tilde{f}_{\min }>0$. Also, $(1-\alpha)^{\alpha}$ is monotonically decreasing with $\alpha$ for $0<\alpha<1$. We therefore get the lower bound

$$
\begin{aligned}
f_{\epsilon}(\alpha) & =(1-\epsilon)^{\alpha}+\epsilon^{\alpha} \tilde{f}(\alpha) \geq(1-\epsilon)+\epsilon^{\alpha} \tilde{f}_{\min } \\
& =1+\epsilon\left(\epsilon^{\alpha-1} \tilde{f}_{\min }-1\right) .
\end{aligned}
$$

Thus, for $\epsilon<\epsilon_{\mathrm{cr}}^{\prime}\left(\alpha_{c}^{\prime}\right):=\left(\tilde{f}_{\min }\right)^{\left[1 /\left(1-\alpha_{c}\right)\right]}$, we have $f_{\epsilon}(\alpha) \geq 1$.

Remark 13. Because of the preceding lemma, in the following, we take the (somewhat arbitrary) choice $\alpha_{c}^{\prime}=$ $1 / 3$ and only consider $\alpha<\alpha_{c}^{\prime}$ as well as values of $\epsilon<\epsilon_{\mathrm{cr}}^{\prime}\left(\alpha_{c}^{\prime}\right)$. Since later we are only interested in arbitrarily small values of $\epsilon$ and $\alpha \leq \delta(\epsilon)$, this is not an obstruction.

Lemma 14. For all $\alpha<\alpha_{c}^{\prime}$ and $\epsilon<\epsilon_{\mathrm{cr}}^{\prime}$, we have $f_{\epsilon}^{\prime}(\alpha) \leq 0$.

Proof.-It follows from the condition that $f_{\epsilon}(\alpha) \geq 1$ for all $\alpha \leq \alpha_{c}^{\prime}$ together with the facts that $f_{\epsilon}(1)=1$, $f_{\epsilon}(0)=Z_{S}$ and that $f_{\epsilon}$ is convex.

We are now in a position to proceed with the proof of the asymptotic concavity. First, we further restrict the values of $\alpha$ by arbitrarily choosing $\alpha_{c}<\alpha_{c}^{\prime}=1 / 3$ and restricting to $\alpha \leq \alpha_{c}$. The reason for this will become clear later in the proof.
Considering Eq. (E10) and using $Z_{S} \geq f_{\epsilon} \geq 1$ as well as Lemma 10, we can now upper bound the second derivative as

$$
\begin{aligned}
S_{\alpha}^{\prime \prime} & \leq-\frac{2}{(1-\alpha)^{2}} \frac{f_{\epsilon}^{\prime}}{f_{\epsilon}}+\frac{1}{(1-\alpha) f_{\epsilon}^{2}}\left(\left(f_{\epsilon}^{\prime}\right)^{2}-f_{\epsilon}^{\prime \prime} f_{\epsilon}\right) \\
& \leq-\frac{2}{\left(1-\alpha_{c}\right)^{2}} \frac{f_{\epsilon}^{\prime}}{f_{\epsilon}}+\frac{1}{Z_{S}^{2}}\left(\left(f_{\epsilon}^{\prime}\right)^{2}-f_{\epsilon}^{\prime \prime} f_{\epsilon}\right) .
\end{aligned}
$$

One might be tempted to use Lemma 10 and simply upper bound the second term by zero, but that bound would be too weak since the first term diverges as $\log (1 / \epsilon)$. Therefore, we now have to do a more detailed calculation. We first compute the derivatives of $f_{\epsilon}$ :

$$
\begin{aligned}
& f_{\epsilon, \alpha}^{\prime}=(1-\epsilon)^{\alpha} \log (1-\epsilon)+\log (\epsilon) \epsilon^{\alpha} \tilde{f}_{\alpha}+\epsilon^{\alpha} \tilde{f}_{\alpha}^{\prime}, \quad \text { (E20) } \\
&\left(f_{\epsilon, \alpha}^{\prime}\right)^{2}=(1-\epsilon)^{2 \alpha} \log (1-\epsilon)^{2}+\log (\epsilon)^{2} \epsilon^{2 \alpha} \tilde{f}_{\alpha}^{2}+\epsilon^{2 \alpha}\left(\tilde{f}_{\alpha}^{\prime}\right)^{2} \\
&+2(1-\epsilon)^{\alpha} \log (1-\epsilon) \log (\epsilon) \epsilon^{\alpha} \tilde{f}_{\alpha} \\
&+2(1-\epsilon)^{\alpha} \log (1-\epsilon) \epsilon^{\alpha} \tilde{f}_{\alpha}^{\prime}+2 \log (\epsilon) \epsilon^{2 \alpha} \tilde{f}_{\alpha} \tilde{f}_{\alpha}^{\prime} .
\end{aligned}
$$

$$
\begin{aligned}
f_{\epsilon, \alpha}^{\prime \prime}= & (1-\epsilon)^{\alpha} \log (1-\epsilon)^{2}+\log (\epsilon)^{2} \epsilon^{\alpha} \tilde{f}_{\alpha} \\
& +2 \log (\epsilon) \epsilon^{\alpha} \tilde{f}_{\alpha}^{\prime}+\epsilon^{\alpha} \tilde{f}_{\alpha}^{\prime \prime},
\end{aligned}
$$

These give

$$
\begin{aligned}
\left(f_{\epsilon, \alpha}^{\prime}\right)^{2}-f_{\epsilon, \alpha}^{\prime \prime} \epsilon^{\alpha} \tilde{f}_{\alpha}= & (1-\epsilon)^{2 \alpha} \log (1-\epsilon)^{2}+\log (\epsilon)^{2} \epsilon^{2 \alpha} \tilde{f}_{\alpha}^{2}+\epsilon^{2 \alpha}\left(\tilde{f}_{\alpha}^{\prime}\right)^{2}+2(1-\epsilon)^{\alpha} \log (1-\epsilon) \log (\epsilon) \epsilon^{\alpha} \tilde{f}_{\alpha} \\
& +2(1-\epsilon)^{\alpha} \log (1-\epsilon) \epsilon^{\alpha} \tilde{f}_{\alpha}^{\prime}+2 \log (\epsilon) \epsilon^{2 \alpha} \tilde{f}_{\alpha} \tilde{f}_{\alpha}^{\prime} \\
& -\epsilon^{\alpha} \tilde{f}_{\alpha}\left((1-\epsilon)^{\alpha} \log (1-\epsilon)^{2}+\log (\epsilon)^{2} \epsilon^{\alpha} \tilde{f}_{\alpha}+2 \log (\epsilon) \epsilon^{\alpha} \tilde{f}_{\alpha}^{\prime}+\epsilon^{\alpha} \tilde{f}_{\alpha}^{\prime \prime}\right) \\
= & \log (1-\epsilon)^{2}(1-\epsilon)^{\alpha}\left((1-\epsilon)^{\alpha}-\epsilon^{\alpha} \tilde{f}_{\alpha}\right)+\epsilon^{2 \alpha}\left(\tilde{f}_{\alpha}^{\prime}\right)^{2} \\
& +2(1-\epsilon)^{\alpha} \log (1-\epsilon) \log (\epsilon) \epsilon^{\alpha} \tilde{f}_{\alpha}+2(1-\epsilon)^{\alpha} \log (1-\epsilon) \epsilon^{\alpha} \tilde{f}_{\alpha}^{\prime}-\epsilon^{2 \alpha} \tilde{f}_{\alpha}^{\prime \prime} \tilde{f}_{\alpha} \\
= & \log (1-\epsilon)^{2}(1-\epsilon)^{\alpha}\left((1-\epsilon)^{\alpha}-\epsilon^{\alpha} \tilde{f}_{\alpha}\right)+2(1-\epsilon)^{\alpha} \log (1-\epsilon) \epsilon^{\alpha}\left(\log (\epsilon) \tilde{f}_{\alpha}+\epsilon^{\alpha} \tilde{f}_{\alpha}^{\prime}\right) \\
& +\epsilon^{2 \alpha}\left(\left(\tilde{f}_{\alpha}^{\prime}\right)^{2}-\tilde{f}_{\alpha}^{\prime \prime} \tilde{f}_{\alpha}\right) .
\end{aligned}
$$

Hence, we have

$$
\begin{aligned}
\left(f_{\epsilon, \alpha}^{\prime}\right)^{2}-f_{\epsilon, \alpha}^{\prime \prime} f_{\alpha}= & -(1-\epsilon)^{\alpha} f_{\epsilon, \alpha}^{\prime \prime}+\log (1-\epsilon)^{2}(1-\epsilon)^{\alpha}\left((1-\epsilon)^{\alpha}-\epsilon^{\alpha} \tilde{f}_{\alpha}\right) \\
& +2(1-\epsilon)^{\alpha} \log (1-\epsilon) \epsilon^{\alpha}\left(\log (\epsilon) \tilde{f}_{\alpha}+\epsilon^{\alpha} \tilde{f}_{\alpha}^{\prime}\right)+\epsilon^{2 \alpha}\left(\left(\tilde{f}_{\alpha}^{\prime}\right)^{2}-\tilde{f}_{\alpha}^{\prime \prime} \tilde{f}_{\alpha}\right) .
\end{aligned}
$$

Note, in particular, that the last term is negative semidefinite because of Lemma 10. 
Let us now also write $0<1 / k_{c}:=\left(1-\alpha_{c}\right)^{2}<1$. Inserting the previous result into Eq. (E18), we then obtain

$$
\begin{aligned}
S_{\alpha}^{\prime \prime} \leq & -2 k_{c} f_{\epsilon, \alpha}^{\prime}+\frac{1}{Z^{2}}\left(\left(f_{\epsilon, \alpha}^{\prime}\right)^{2}-f_{\epsilon, \alpha}^{\prime \prime} f_{\alpha}\right) \\
\leq & -2 k_{c} f_{\epsilon, \alpha}^{\prime}+\frac{1}{Z^{2}}\left[-(1-\epsilon) f_{\epsilon, \alpha}^{\prime \prime}+\log (1-\epsilon)^{2}(1-\epsilon)^{\alpha}\left((1-\epsilon)^{\alpha}-\epsilon^{\alpha} \tilde{f}_{\alpha}\right)\right. \\
& \left.+2(1-\epsilon)^{\alpha} \log (1-\epsilon) \epsilon^{\alpha}\left(\log (\epsilon) \tilde{f}_{\alpha}+\epsilon^{\alpha} \tilde{f}_{\alpha}^{\prime}\right)\right] \\
\leq & -2 k_{c} f_{\epsilon, \alpha}^{\prime}+\frac{1}{Z^{2}}\left[-(1-\epsilon) f_{\epsilon, \alpha}^{\prime \prime}+\log (1-\epsilon)^{2}\left(1-(1-\epsilon) \epsilon \tilde{f}_{\alpha}\right)\right. \\
& \left.+2 \log (1-\epsilon)\left(\log (\epsilon) \tilde{f}_{\alpha}+\epsilon^{2 \alpha}(1-\epsilon)^{\alpha} \tilde{f}_{\alpha}^{\prime}\right)\right]
\end{aligned}
$$

We now lower bound $f_{\epsilon}^{\prime}$ and $f_{\epsilon}^{\prime \prime}$ as

$$
\begin{gathered}
f_{\epsilon, \alpha}^{\prime} \geq \log (1-\epsilon)+\log (\epsilon) \tilde{f}_{\alpha}+\tilde{f}_{\alpha}^{\prime} \\
f_{\epsilon, \alpha}{ }^{\prime \prime} \geq(1-\epsilon) \log (1-\epsilon)^{2}+\log (\epsilon)^{2} \epsilon^{\alpha_{c}} \tilde{f}_{\alpha}+2 \log (\epsilon) \epsilon^{\alpha} \tilde{f}_{\alpha}^{\prime}+\epsilon \tilde{f}_{\alpha}^{\prime \prime} .
\end{gathered}
$$

Note that we cannot easily bound the terms involving $\tilde{f}_{\alpha}^{\prime}$ since we do not know the sign of $\tilde{f}_{\alpha}^{\prime}$. However, we emphasize again that $\tilde{f}$ is independent of $\epsilon$ and can hence essentially be treated as constant. Putting in the bounds then yields

$$
\begin{aligned}
S_{\alpha}^{\prime \prime} \leq & -2 k_{c}\left(\log (1-\epsilon)+\log (\epsilon) \tilde{f}_{\alpha}+\tilde{f}_{\alpha}^{\prime}\right)-\frac{1-\epsilon}{Z^{2}}\left((1-\epsilon) \log (1-\epsilon)^{2}+\log (\epsilon)^{2} \epsilon^{\alpha_{c}} \tilde{f}_{\alpha}+2 \log (\epsilon) \epsilon^{\alpha} \tilde{f}_{\alpha}^{\prime}+\epsilon \tilde{f}_{\alpha}^{\prime \prime}\right) \\
& +\frac{1}{Z^{2}}\left[\log (1-\epsilon)^{2}\left(1-(1-\epsilon) \epsilon \tilde{f}_{\alpha}\right)+2 \log (1-\epsilon)\left(\log (\epsilon) \tilde{f}_{\alpha}+\epsilon^{2 \alpha}(1-\epsilon)^{\alpha} \tilde{f}_{\alpha}^{\prime}\right)\right] \\
= & \log (\epsilon) \tilde{f}_{\alpha}\left(\frac{2}{Z^{2}} \log (1-\epsilon)-\frac{1-\epsilon}{Z^{2}} 2 \epsilon^{\alpha} \frac{\tilde{f}_{\alpha}^{\prime}}{\tilde{f}_{\alpha}}-\frac{1-\epsilon}{Z^{2}} \log (\epsilon) \epsilon^{\alpha_{c}}-\frac{2}{\left(1-\alpha_{c}\right)^{2}}\right) \\
& +\log (1-\epsilon)\left(-\frac{2}{\left(1-\alpha_{c}\right)^{2}}-\frac{(1-\epsilon)^{2}}{Z^{2}} \log (1-\epsilon)+\frac{1-(1-\epsilon) \epsilon \tilde{f}_{\alpha}}{Z^{2}} \log (1-\epsilon)+\frac{2}{Z^{2}} \epsilon^{2 \alpha}(1-\epsilon)^{\alpha} \tilde{f}_{\alpha}^{\prime}\right) \\
& -\frac{2}{\left(1-\alpha_{c}\right)^{2}} \tilde{f}_{\alpha}^{\prime}-\frac{(1-\epsilon) \epsilon}{Z^{2}} \tilde{f}_{\alpha}^{\prime \prime} \\
\leq & \log (\epsilon) \tilde{f}_{\alpha}\left(\frac{2}{Z^{2}} \log (1-\epsilon)-\frac{1-\epsilon}{Z^{2}} 2 \epsilon^{\alpha} \frac{\tilde{f}_{\alpha}^{\prime}}{\tilde{f}_{\alpha}}-\frac{1-\epsilon}{Z^{2}} \log (\epsilon) \epsilon^{\alpha_{c}}-\frac{2}{\left(1-\alpha_{c}\right)^{2}}\right)+M\left(\epsilon, H, \beta, \rho^{\perp}\right)-K\left(\alpha_{c}, H, \beta, \rho^{\perp}\right),
\end{aligned}
$$

where $M$ goes to zero as $\epsilon$ goes to zero and $K$ is independent of $\epsilon$. Also note that $M$ is bounded and independent of $\alpha$ (because of the boundedness of $\tilde{f}_{\alpha}$ and its derivatives). Let us define $m\left(\alpha_{c}\right)=\max _{\alpha \leq \alpha_{c}} \tilde{f}_{\alpha}^{\prime} / \tilde{f}_{\alpha}$. Since $\alpha_{c}<1 / 2$, we can simplify the bound to

$S_{\alpha}^{\prime \prime} \leq \log (\epsilon) \frac{\tilde{f}_{\alpha}}{Z^{2}}\left(2 \log (1-\epsilon)-2(1-\epsilon) \epsilon^{\alpha} m\left(\alpha_{c}\right)-(1-\epsilon) \log (\epsilon) \epsilon^{\alpha_{c}}-8 Z^{2}\right)+M\left(\epsilon, H, \beta, \rho^{\perp}\right)-K\left(\alpha_{c}, H, \beta, \rho^{\perp}\right)$.

Clearly, $S_{\alpha}^{\prime \prime}$ can be made negative by taking $\epsilon$ and $\alpha_{c}$ arbitrarily small since the dominant term in the brackets goes as $-\log (\epsilon)$. However, since our objective is to upper bound $S_{\alpha}$ by $\mathcal{V}_{\beta}\left(\rho_{\epsilon}, H_{S}\right) \alpha$ for all $\alpha \leq \alpha_{c}$, we also need $\mathcal{V}_{\beta}\left(\rho_{\epsilon}, H_{S}\right) \alpha_{c} \geq$ $\log Z_{S}$ and hence $\alpha_{c} \geq \log \left(Z_{S}\right) / \mathcal{V}_{\beta}\left(\rho_{\epsilon}, H_{S}\right)$. Hence, we choose $\alpha_{c}=\delta(\epsilon)=\log Z_{S} / \mathcal{V}_{\beta}\left(\rho_{\epsilon}, H_{S}\right)$ and hope for the best. The vacancy is given by 


$$
\begin{aligned}
\mathcal{V}_{\beta}\left(\rho_{\epsilon}, H_{S}\right)= & -\log (1-\epsilon) \frac{1}{Z_{S}}+\frac{Z_{S}-1}{Z_{S}} \log (1 / \epsilon) \\
& +C_{1}\left(\rho^{\perp}, \beta, H_{S}\right),
\end{aligned}
$$

where $C_{1}$ does not depend on $\epsilon$. Hence,

$$
\begin{aligned}
\lim _{\epsilon \rightarrow 0} \epsilon^{\delta(\epsilon)} & =\frac{1}{Z_{S}^{\left[\left(Z_{S}\right) / Z_{S}-1\right]}} \quad \text { and } \\
\lim _{\epsilon \rightarrow 0}-(1-\epsilon) \log (\epsilon) \epsilon^{\delta(\epsilon)} & =+\infty .
\end{aligned}
$$

Since the other terms in the first brackets in $S_{\alpha}^{\prime \prime}$ go to zero as $\epsilon \rightarrow 0$ and $K$ is independent of $\epsilon$, we can find a finite $\epsilon_{c r}$ such that

$$
S_{\alpha}^{\prime \prime} \leq 0, \alpha \leq \delta\left(\epsilon_{c r}\right)
$$

This finishes the proof.

\section{APPENDIX F: EXACTLY CONSERVED CATALYSTS}

In this section, we analyze the scenario where the catalyst always has to be returned without any error. In other words, the cooling protocol considers a process like the one described in Sec. II but taking $\epsilon=0$. First, note that the vacancy is also automatically a monotone in this setting since we are considering a subset of free operations. Hence, the inequality

$$
V_{\beta}\left(\rho_{R}, H_{R}\right) \geq V_{\beta}\left(\rho_{S}, H_{S}\right)
$$

is also a necessary condition for this set of free operations. In the following, we consider, for simplicity, only the case where the target system is thermal, $\rho_{S}=\omega_{\beta_{S}}\left(H_{S}\right)$.

We now prove the following theorem, which provides a sufficient condition for cooling and which coincides with that in our general Theorem 2 up to a multiplicative factor.

Theorem 15 (Sufficient condition under exact catalysis). Assume thermal operations with exact catalysts. Then, for every choice of $\beta$ and $H_{S}$, there is a critical $\beta_{\mathrm{cr}}>0$ such that for any $\beta_{S}>\beta_{\text {cr }}$ and full-rank resource $\left(\rho_{R}, H_{R}\right)$ (diagonal in the energy eigenbasis), the condition

$$
\begin{gathered}
\mathcal{V}_{\beta}\left(\rho_{R}, H_{R}\right)-K\left(\beta_{S}, \beta, \rho_{R}, H_{R}, H_{S}\right) \\
\geq r(\beta, H) \mathcal{V}_{\beta}\left(\omega_{\beta_{S}}\left(H_{S}\right), H_{S}\right)
\end{gathered}
$$

is sufficient for cooling. The positive-semidefinite function $K$ is identical to that in Theorem 2, and the constant $r\left(\beta, H_{S}\right)$ is independent of $\rho_{R}, H_{R}$, and $\beta_{S}$ and given by

$$
r\left(\beta, H_{S}\right)=1+2 \frac{E_{\max }-E_{\beta}}{E_{\beta}},
$$

where $E_{\max }$ is the largest eigenvalue of $H_{S}$ and we assume that the ground-state energy of $H_{S}$ is zero.

Before presenting the proof, let us briefly discuss the implications that the correction given by $r\left(\beta, H_{S}\right)$ has over the sufficient condition Theorem 2. This is better explained if we look at the scaling results of Sec. IV. There, we showed that the sufficient condition of Theorem 2 provides an upper bound on the number of copies of a resource that are sufficient to implement a cooling process, as given by $n^{\text {suff }}$ in Eq. (22). The sufficient condition laid out in Theorem 15 implies simply that we need $r$ times more systems to implement the cooling protocol, where $r=r(\beta, H)$. Note importantly that $r$ does not depend on the final temperature, so employing $r\left(\beta, H_{S}\right) \times n^{\text {suff }}$ is always sufficient for cooling. We emphasize that we believe that the factor $r\left(\beta, H_{S}\right)$ can be made much closer to 1 by more elaborate proof techniques, but we leave this as an open problem.

Proof.-It was shown in Ref. [34] that a transition $\rho \rightarrow \rho^{\prime}$ between two diagonal states is possible with exact preservation of the catalyst if and only if the Renyi divergences

$$
S_{\alpha}\left(\rho \| \omega_{\beta}(H)\right)=\frac{\operatorname{sign}(\alpha)}{\alpha-1} \log \operatorname{Tr}\left(\rho^{\alpha} \| \omega_{\beta}(H)^{1-\alpha}\right)
$$

do not increase for all $\alpha \in(-\infty,+\infty)$. The sufficient condition in Theorem 2 covers all $\alpha \geq 0$. We thus have to check that we can fulfill all the inequalities for $\alpha<0$ using the multiplicative factor $r\left(\beta, H_{S}\right)$. To do this, we provide new lower and upper bounds for the Renyi divergences for negative $\alpha$. We begin with a lower bound. Consider any state $\rho$ with eigenvalues $p_{i}$ in the energy eigenbasis. Then, we have

$$
S_{-|\alpha|}\left(\rho \| \omega_{\beta}(H)\right)=\frac{1}{|\alpha|+1} \log \left(\sum_{i} p_{i}^{-|\alpha|} w_{i}^{1+|\alpha|}\right),
$$

where $w_{i}=\mathrm{e}^{-\beta E_{i}} / Z_{\beta}$ are the eigenvalues of the thermal state. Using concavity of the logarithm, we can bound this as

$$
\begin{aligned}
S_{-|\alpha|}\left(\rho \| \omega_{\beta}(H)\right) & \geq \frac{1}{|\alpha|+1} \sum_{i} w_{i} \log \left(p_{i}^{-|\alpha|} w_{i}^{|\alpha|}\right) \\
& =\frac{|\alpha|}{|\alpha|+1} \sum_{i}\left(w_{i} \log \left(w_{i}\right)-w_{i} \log \left(p_{i}\right)\right) \\
& =\frac{|\alpha|}{|\alpha|+1} V_{\beta}(\rho, H)
\end{aligned}
$$

We can thus lower bound all the Renyi divergences for negative $\alpha$ by a simple function. Later, we apply this bound to the resource. 
We now derive a similar upper bound for the target system, i.e., assuming a system in a thermal state. First, we rewrite the Renyi divergences as

$$
\begin{aligned}
& S_{-|\alpha|}\left(\omega_{\beta_{S}}\left(H_{S}\right) \| \omega_{\beta}\left(H_{S}\right)\right) \\
& =\frac{|\alpha|}{|\alpha|+1} \log \left(Z_{\beta_{S}}\right)-\log \left(Z_{\beta}\right) \\
& \quad+\frac{1}{1+|\alpha|} \log \operatorname{Tr}\left(\mathrm{e}^{\left(\beta_{S}-\beta\right)|\alpha| H_{S}} \mathrm{e}^{-\beta H_{S}}\right),
\end{aligned}
$$

which can be verified by direct calculation. We now use the $\log$-sum inequality. It states that for any two sets of $d$ nonnegative numbers $\left\{a_{i}\right\}$ and $\left\{b_{i}\right\}$, we have

$$
\log \frac{a}{b} \leq \sum_{i} \frac{a_{i}}{a} \log \frac{a_{i}}{b_{i}}
$$

with $a=\sum_{i} a_{i}$ and $b=\sum_{i} b_{i}$. Let $E_{i}$ be the energy eigenvalues of $H_{S}$. Then, we set

$a_{i}=\mathrm{e}^{\left(\beta_{S}-\beta\right)|\alpha| E_{i}} \mathrm{e}^{-\beta E_{i}}=\mathrm{e}^{-\tilde{\beta}(\alpha) E_{i}}, \quad b_{i}=\frac{\mathrm{e}^{-\beta E_{i}}}{Z_{\beta}}$,

where $\tilde{\beta}(\alpha):=\beta-\left(\beta_{S}-\beta\right)|\alpha|$. Using the log-sum inequality, we then obtain

$$
\begin{aligned}
S_{-|\alpha|}\left(\omega_{\beta_{S}}\left(H_{S}\right) \| \omega_{\beta}\left(H_{S}\right)\right) & \leq \frac{|\alpha|}{|\alpha|+1} \log \left(Z_{\beta_{S}}\right)-\log \left(Z_{\beta}\right)+\frac{1}{1+|\alpha|} \sum_{i} \frac{\mathrm{e}^{-\tilde{\beta}(\alpha) E_{i}}}{Z_{\tilde{\beta}(\alpha)}} \log \left(\mathrm{e}^{\left(\beta_{S}-\beta\right)|\alpha| E_{i}} Z_{\beta}\right) \\
& =\frac{|\alpha|}{|\alpha|+1}\left(\log \left(Z_{\beta_{S}}\right)-\log \left(Z_{\beta}\right)\right)+\frac{|\alpha|}{|\alpha|+1}\left(\beta_{S}-\beta\right) \sum_{i} \frac{\mathrm{e}^{-\tilde{\beta}(\alpha) E_{i}}}{Z_{\tilde{\beta}(\alpha)}} E_{i} .
\end{aligned}
$$

Denoting by $E_{\max }$ the maximum energy, we then get the bound

$$
S_{-|\alpha|}\left(\omega_{\beta_{S}}\left(H_{S}\right) \| \omega_{\beta}\left(H_{S}\right)\right) \leq \frac{|\alpha|}{|\alpha|+1}\left(\log \left(Z_{\beta_{S}}\right)-\log \left(Z_{\beta}\right)+\left(\beta_{S}-\beta\right) E_{\max }\right) .
$$

Let us recall from Eq. (24) that for thermal states, the vacancy can be expressed as a function of the nonequilibrium free energy as

$$
\mathcal{V}_{\beta}\left(\omega_{\beta_{S}}\left(H_{S}\right), H_{S}\right)=\beta_{S} \Delta F_{\beta_{S}}\left(\omega_{\beta}(H), H\right)=\beta_{S} E_{\beta}-S_{\beta}+\log \left(Z_{\beta_{S}}\right),
$$

where $E_{\beta}$ and $S_{\beta}$ denote the thermal energy expectation value and von Neumann entropy at inverse temperature $\beta$. Using this result, together with $-\log Z_{\beta}=\beta E_{\beta}-S_{\beta}$, we can rewrite the upper bound on the Renyi divergences as

$$
\begin{aligned}
S_{-|\alpha|}\left(\omega_{\beta_{S}}\left(H_{S}\right) \| \omega_{\beta}\left(H_{S}\right)\right) & \leq \frac{|\alpha|}{|\alpha|+1}\left(\mathcal{V}_{\beta}\left(\omega_{\beta_{S}}\left(H_{S}\right), H_{S}\right)\left[1+\frac{E_{\max }-E_{\beta}}{\Delta F_{\beta_{S}}\left(\omega_{\beta}(H), H\right)}\right]-\beta\left(E_{\max }-E_{\beta}\right)\right) \\
& \leq \frac{|\alpha|}{|\alpha|+1} \mathcal{V}_{\beta}\left(\omega_{\beta_{S}}\left(H_{S}\right), H_{S}\right)\left[1+\frac{E_{\max }-E_{\beta}}{\Delta F_{\beta_{S}}\left(\omega_{\beta}(H), H\right)}\right] .
\end{aligned}
$$

Since we have

$$
\Delta F_{\beta_{S}}\left(\omega_{\beta}(H), H\right)=E_{\beta}-E_{\beta_{S}}-\frac{1}{\beta_{S}}\left(S_{\beta}-S_{\beta_{S}}\right),
$$

it is always possible to find a critical inverse temperature $\beta_{S}^{\prime}$ such that $\Delta F_{\beta_{S}}\left(\omega_{\beta}(H), H\right) \geq E_{\beta} / 2$ for all $\beta_{S}>\beta_{S}^{\prime}$. Then, for all $\beta_{S}$ larger than this critical temperature, we can bound the Renyi divergences as

$$
S_{-|\alpha|}\left(\omega_{\beta_{S}}\left(H_{S}\right) \| \omega_{\beta}\left(H_{S}\right)\right) \leq \frac{|\alpha|}{|\alpha|+1} \mathcal{V}_{\beta}\left(\omega_{\beta_{S}}\left(H_{S}\right), H_{S}\right)\left[1+2 \frac{E_{\max }-E_{\beta}}{E_{\beta}}\right]=: \frac{|\alpha|}{|\alpha|+1} \mathcal{V}_{\beta}\left(\omega_{\beta_{S}}\left(H_{S}\right), H_{S}\right) r\left(\beta, H_{S}\right) .
$$

Using the lower bound (F6) for the resource, we then find that the inequalities for negative $\alpha$ are fulfilled if 


$$
\frac{|\alpha|}{|\alpha|+1} \mathcal{V}_{\beta}\left(\rho_{R}, H_{R}\right) \geq \frac{|\alpha|}{|\alpha|+1} \mathcal{V}_{\beta}\left(\omega_{\beta_{S}}\left(H_{S}\right), H_{S}\right) r\left(\beta, H_{S}\right) .
$$

Canceling the prefactors, the sufficient condition for negative values of $\alpha$ is the inequality

$$
\mathcal{V}_{\beta}\left(\rho_{R}, H_{R}\right) \geq \mathcal{V}_{\beta}\left(\omega_{\beta_{S}}\left(H_{S}\right), H_{S}\right) r\left(\beta, H_{S}\right) .
$$

Combining this with the sufficient condition for positive $\alpha$, which is the sufficient condition provided by Theorem 2, then yields the claimed sufficient condition in the theorem.

\section{Catalysts can always be chosen with full rank}

Before finishing this section, let us point out that in the case of exact catalysis, one can always choose the catalyst to have full rank. In other words, the actual implementation of the cooling protocol, which is guaranteed to exist under the conditions of Theorem 15, never requires that we employ a catalyst that is not full rank.

To see this, consider a bipartite system with noninteracting Hamiltonian $H_{1}+H_{2}$. Then, consider the initial state $\rho_{S B} \otimes \sigma_{C}$ and apply an energy-preserving unitary operation $U$ that results in the state $\rho_{S B C}^{\prime}$ with $\rho_{C}^{\prime}=\sigma_{C}$. Here, we imagine that $\rho_{S B}$ also includes the state of the heat bath, and thus there can be a buildup of correlations between the catalyst and $\rho_{S B}$. Furthermore, assume that $\rho_{S B}$ has full rank and that $\sigma_{C}$ is supported only on a subspace $P \subset \mathcal{H}_{2}$ with complement $Q=\mathbf{1}-P$ (we identify the vector space and the projector on the space). Thus, $P=\sum_{j}|j\rangle\langle j|$, where the sum is over the eigenstates of $\sigma_{C}$. Let the spectrum of $\rho_{S B}$ and $\sigma_{C}$ be $\left\{p_{\alpha}\right\}$ and $\left\{q_{j}\right\}$, respectively. Then, the final state

$$
\rho_{\mathrm{SBC}}^{\prime}=\sum_{\alpha, j} p_{\alpha} q_{j} U|\alpha\rangle\langle\alpha|\otimes| j\rangle\langle j| U^{\dagger}
$$

is a convex sum of the positive semidefinite operators $U|\alpha\rangle\langle\alpha|\otimes| j\rangle\langle j| U^{\dagger}$. The sum has support only within $1 \otimes P$ since otherwise the reduced state $\rho_{C}^{\prime}$ would also be supported outside of $P$. Hence, every summand is also supported within $1 \otimes P$. Using $1 \otimes P=\sum_{\alpha, j}|\alpha\rangle\langle j|\otimes| j\rangle\langle j|$, we then obtain

$$
(\mathbf{1} \otimes Q) U(\mathbf{1} \otimes P) U^{\dagger}=\mathbf{1} \otimes Q \sum_{\alpha, j} U|\alpha\rangle\langle j|\otimes| j\rangle\langle j| U^{\dagger}=0 .
$$

In other words, we have $(\mathbf{1} \otimes Q) U(\mathbf{1} \otimes P)=0$ and, by a similar calculation, also $(\mathbf{1} \otimes P) U(\mathbf{1} \otimes Q)=0$. Thus, the unitary $U$ is block diagonal. In particular, the operator $V=$ $(\mathbf{1} \otimes P) U(\mathbf{1} \otimes P)$ considered as an operator on the Hilbert space $\mathcal{H}_{1} \otimes P$ is unitary. Since $U$ is energy preserving by assumption, we can deduce that $P=\operatorname{span}\left\{\left|E_{j}\right\rangle\right\}$ for some subset of energy eigenstates $\left|E_{j}\right\rangle$ of the Hamiltonian $\mathrm{H}_{2}$ of the catalyst.

Then, $V$ commutes with the Hamiltonian $H_{1}+\left.H_{2}\right|_{P}$, where $\left.H_{2}\right|_{P}$ denotes the Hamiltonian of the catalyst, but restricted to the subspace $P$.

We can thus obtain an equivalent catalyst with full rank and a corresponding thermal operation by restricting $\sigma_{C}$ and $H_{2}$ to the subspace $P$ on which $\sigma_{C}$ has full rank and using the thermal operation defined by $V$ :

$$
\left.V \rho_{S B} \otimes \sigma_{C}\right|_{P} V^{\dagger}=\left.\rho_{\mathrm{SBC}}^{\prime}\right|_{1 \otimes P} .
$$

In particular, note that the above analysis also shows that, in the case of exact catalysis, pure catalysts are useless: If a transition can be done with a pure catalyst, it can also be done without a catalyst.

\section{APPENDIX G: APPROXIMATE CATALYSIS}

In this article, we have assumed that catalysts are returned arbitrarily close to their initial state (or, exactly, in the last section). Here, we discuss possible relaxations of this assumption to include approximate catalysts.

First, we note that the problem of allowing for finite errors - in some suitable measure - between the initial and final states of the catalyst is a delicate one, especially in the context of the third law of thermodynamics. The challenge is caused by the fact that the statement of the unattainability principle is not stable under arbitrarily good approximations: It compares the case where the state of the target system is exactly the ground state with the case of approximating the ground state to arbitrary precision. In the former case, infinite resources are needed, while in the latter case, finite resources are needed (however, diverging with the approximation precision). This is the ultimate reason why a discontinuous measure of resources (like the vacancy) is necessary to capture the third law in the resource theoretic setting.

With this in mind, let us discuss the problem of approximate catalysts. If one demands that the catalyst is returned in approximately the same state, it is crucial how one measures "approximately." In the context of thermal operations, this problem has been studied in Refs. [34,54]. It has been shown in Ref. [34] that if one requires only that the catalyst is returned up to an arbitrarily small but fixed error in trace distance, any transition can be implemented using a thermal operation to arbitrary precisionwithout any resource. In particular, this implies that perfect cooling can be achieved without using any resource state. Therefore, it is clear that stronger conditions are necessary so as not to trivialize the problem of cooling.

A second way to define approximate catalysts is to require that the catalyst is returned up to an error $\epsilon / \log (d)$ in trace distance, where $d$ is the dimension of the catalyst, and $\epsilon>0$ is arbitrarily small but fixed for all catalysts. 
Intuitively, this definition requires that the error is small even when multiplied by the number of particles in the catalyst. In this case, transitions can be implemented to arbitrary precision if the nonequilibrium free energy decreases [34]. This would lead to requiring a constant amount of resources to cool a given system to arbitrary low temperatures-hence, the unattainability principle is also violated in this case.

Because of the arguments above, it seems that allowing for a finite error-measured in trace distance-in the catalyst seems to be too forgiving. However, Ref. [34] also hints at a solution to this problem: One should measure the error in terms of a quantity that is meaningful for the problem at hand. In Ref. [34], the authors consider the problem of work extraction and demand in turn that the catalyst is returned with approximately the same "work distance," where the work distance measures the potential of one state to produce work. In our case, we are concerned with the task of low-temperature cooling. Indeed, the vacancy itself plays the role of the cooling potential since the limitations for low-temperature cooling of Theorem 1 are expressed in terms of the vacancy. We can thus require that the catalyst has to be returned with a vacancy that differs only by an amount $\epsilon$ from the initial vacancy. If we adopt this definition of approximate catalysts, the general necessary condition (11) is modified only slightly. This can be seen in the following way. First, note that this notion requires that catalysts all have finite vacancy; i.e., they must have full rank. In this case, we can simply evaluate the vacancy of the resource, system, and target before and after the cooling protocol has been applied. Let us assume that the initial state of the catalyst is $\sigma$, while the final state is $\sigma^{\prime}$. Since the vacancy is an additive monotone of thermal operations and vanishes on thermal states, we then obtain

$$
\begin{aligned}
& \mathcal{V}_{\beta}\left(\rho_{R} \otimes \omega_{\beta}\left(H_{S}\right) \otimes \sigma, H_{R}+H_{S}+H_{C}\right) \\
& \quad=\mathcal{V}_{\beta}\left(\rho_{R}, H_{R}\right)+\mathcal{V}_{\beta}\left(\sigma, H_{C}\right) \\
& \quad \geq \mathcal{V}_{\beta}\left(\omega_{\beta}\left(H_{R}\right) \otimes \rho_{S} \otimes \sigma^{\prime}, H_{R}+H_{S}+H_{C}\right) \\
& \quad=V_{\beta}\left(\rho_{S}, H_{S}\right)+\mathcal{V}_{\beta}\left(\sigma^{\prime}, H_{C}\right) .
\end{aligned}
$$

Hence, we obtain, as a new necessary condition,

$$
\mathcal{V}_{\beta}\left(\rho_{R}, H_{R}\right)+\epsilon \geq V_{\beta}\left(\rho_{S}, H_{S}\right),
$$

with $\epsilon=\mathcal{V}_{\beta}\left(\sigma, H_{C}\right)-\mathcal{V}_{\beta}\left(\sigma^{\prime}, H_{C}\right)$ being the error in the catalyst measured by the vacancy.

Thus, the necessary condition and hence the quantitative unattainability principle is stable under approximate catalysts if defined consistently: allowing a fixed but small error measured by the vacancy difference.

It seems plausible that under this definition of catalysts, the sufficient condition in Theorem 2 also simplifies to Eq. (G3) for arbitrary resources-at least for low enough target temperatures. However, proving this statement rigorously seems to require further technical innovations beyond the scope of this work. We therefore leave this as an open problem.

[1] W. Nernst, Über die Berechnung chemischer Gleichgewichte aus thermischen Messungen, Nachr. Kgl. Ges. Wiss. Goett. 1, 1 (1906).

[2] W. Nernst, Über die Beziehung zwischen Wärmeentwicklung und maximaler Arbeit bei kondensierten Systemen, Ber. Kgl. Pr. Akad. Wiss. 52, 933 (1906).

[3] L. Masanes and J. Oppenheim, A General Derivation and Quantification of the Third Law of Thermodynamics, Nat. Commun. 8, 14538 (2017).

[4] J. Scharlau and M. P. Mueller, Quantum Horn's Lemma, Finite Heat Baths, and the Third Law of Thermodynamics, arXiv:1605.06092.

[5] R. Silva, G. Manzano, P. Skrzypczyk, and N. Brunner, Performance of Autonomous Quantum Thermal Machines: Hilbert Space Dimension as a Thermodynamical Resource, Phys. Rev. E 94, 032120 (2016).

[6] C. Di Franco and M. Paternostro, A No-Go Result on the Purification of Quantum States, Sci. Rep. 3, 1387 (2013).

[7] L.-A. Wu, D. Segal, and P. Brumer, No-Go Theorem for Ground State Cooling Given Initial System-Thermal Bath Factorization, Sci. Rep. 3, 1824(2013).

[8] F. Ticozzi and L. Viola, Quantum Resources for Purification and Cooling: Fundamental Limits and Opportunities, Sci. Rep. 4, 5192 (2014).

[9] L. J. Schulman and U. V. Vazirani, Molecular Scale Heat Engines and Scalable Quantum Computation, Proceedings of the 31st Annual ACM Symposium on Theory of Computing, Atlanta, Georgia (ACM, New York, 1999), pp. 322-329.

[10] P. O. Boykin, T. Mor, V. Roychowdhury, F. Vatan, and R. Vrijen, Algorithmic Cooling and Scalable NMR Quantum Computers, Proc. Natl. Acad. Sci. U.S.A. 99, 3388 (2002).

[11] L. J. Schulman, T. Mor, and Y. Weinstein, Physical Limits of Heat-Bath Algorithmic Cooling, Phys. Rev. Lett. 94, 120501 (2005).

[12] A. E. Allahverdyan, K. V. Hovhannisyan, D. Janzing, and G. Mahler, Thermodynamic Limits of Dynamic Cooling, Phys. Rev. E 84, 041109 (2011).

[13] S. Raeisi and M. Mosca, Asymptotic Bound for Heat-Bath Algorithmic Cooling, Phys. Rev. Lett. 114, 100404 (2015).

[14] A.E. Allahverdyan, K. Hovhannisyan, and G. Mahler, Optimal Refrigerator, Phys. Rev. E 81, 051129 (2010).

[15] N. Linden, S. Popescu, and P. Skrzypczyk, How Small Can Thermal Machines Be? The Smallest Possible Refrigerator, Phys. Rev. Lett. 105, 130401 (2010).

[16] P. Skrzypczyk, N. Brunner, N. Linden, and S. Popescu, The Smallest Refrigerators Can Reach Maximal Efficiency, J. Phys. A 44, 492002 (2011).

[17] A. Levy, R. Alicki, and R. Kosloff, Quantum Refrigerators and the Third Law of Thermodynamics, Phys. Rev. E 85, 061126 (2012). 
[18] A. Mari and J. Eisert, Cooling by Heating: Very Hot Thermal Light Can Significantly Cool Quantum Systems, Phys. Rev. Lett. 108, 120602 (2012).

[19] R. Silva, P. Skrzypczyk, and N. Brunner, Small Quantum Absorption Refrigerator with Reversed Couplings, Phys. Rev. E 92, 012136 (2015).

[20] P. P. Hofer, M. Perarnau-Llobet, J. B. Brask, R. Silva, M. Huber, and N. Brunner, Autonomous Quantum Refrigerator in a Circuit QED Architecture Based on a Josephson Junction, Phys. Rev. B 94, 235420 (2016).

[21] B. Cleuren, B. Rutten, and C. Van den Broeck, Cooling by Heating: Refrigeration Powered by Photons, Phys. Rev. Lett. 108, 120603 (2012).

[22] A. E. Allahverdyan, K. V. Hovhannisyan, and G. Mahler, Comment on "Cooling by Heating: Refrigeration Powered by Photons," Phys. Rev. Lett. 109, 248903 (2012).

[23] A. Levy, R. Alicki, and R. Kosloff, Comment on "Cooling by Heating: Refrigeration Powered by Photons," Phys. Rev. Lett. 109, 248901 (2012).

[24] O. Entin-Wohlman and Y. Imry, Comment on "Cooling by Heating: Refrigeration Powered by Photons," Phys. Rev. Lett. 112, 048901 (2014).

[25] B. Cleuren, B. Rutten, and C. Van den Broeck et al. Reply to "Comment on 'Cooling by Heating: Refrigeration Powered by Photons,'” Phys. Rev. Lett. 109, 248902 (2012).

[26] M. Kol, D. Gelbwaser-Klimovsky, R. Alicki, and G. Kurizki, Quantum Bath Refrigeration Towards Absolute Zero: Challenging the Unattainability Principle, Phys. Rev. Lett. 109, 090601 (2012).

[27] R. Kosloff, Quantum Thermodynamics: A Dynamical Viewpoint, Entropy 15, 2100 (2013).

[28] N. Freitas and J. P. Paz, Fundamental Limits for Cooling of Linear Quantum Refrigerators, Phys. Rev. E 95, 012146 (2017).

[29] D. Janzing, P. Wocjan, R. Zeier, R. Geiss, and Th. Beth, Thermodynamic Cost of Reliability and Low Temperatures: Tightening Landauer's Principle and the Second Law, Int. J. Theor. Phys. 39, 2717 (2000).

[30] M. Horodecki and J. Oppenheim, Fundamental Limitations for Quantum and Nanoscale Thermodynamics, Nat. Commun. 4, 2059 (2013).

[31] G. Gour, M. P. Mller, V. Narasimhachar, R. W. Spekkens, and N. Y. Halpern, The Resource Theory of Informational Nonequilibrium in Thermodynamics, Phys. Rep. 583, 1 (2015).

[32] N. Y. Halpern, Beyond Heat Baths II: Framework for Generalized Thermodynamic Resource Theories, arXiv: 1409.7845.

[33] N. Y. Halpern and J. M. Renes, Beyond Heat Baths: Generalized Resource Theories for Small-Scale Thermodynamics, Phys. Rev. E 93, 022126 (2016).

[34] F. G. S. L. Brandao, M. Horodecki, N. H. Y. Ng, J. Oppenheim, and S. Wehner, The Second Laws of Quantum Thermodynamics, Proc. Natl. Acad. Sci. U.S.A. 112, 3275 (2015).

[35] P. Faist, F. Dupuis, J. Oppenheim, and R. Renner, The Minimal Work Cost of Information Processing, Nat. Commun. 6, 7669 (2015).
[36] N. Y. Halpern, A. J. P. Garner, O. C. O. Dahlsten, and V. Vedral, Introducing One-Shot Work into Fluctuation Relations, New J. Phys. 17, 095003 (2015).

[37] J. Aberg, Truly Work-like Work Extraction via a Single-Shot Analysis, Nat. Commun. 4, 1925 (2013).

[38] R. Gallego, J. Eisert, and H. Wilming, Thermodynamic Work from Operational Principles, New J. Phys. 18, 103017 (2016).

[39] 1. M. Alhambra, L. Masanes, J. Oppenheim, and C. Perry, The Second Law of Quantum Thermodynamics as an Equality, Phys. Rev. X 6, 041017 (2016).

[40] J. Aberg, Fully Quantum Fluctuation Theorems, arXiv: 1601.01302.

[41] L. del Rio, J. Aberg, R. Renner, O. Dahlsten, and V. Vedral, The Thermodynamic Meaning of Negative Entropy, Nature (London) 474, 61 (2011).

[42] J. Aberg, Catalytic Coherence, Phys. Rev. Lett. 113, 150402 (2014).

[43] P. Faist, J. Oppenheim, and R. Renner, Gibbs-Preserving Maps Outperform Thermal Operations in the Quantum Regime, New J. Phys. 17, 043003 (2015).

[44] M. Lostaglio, K. Korzekwa, D. Jennings, and T. Rudolph, Quantum Coherence, Time-Translation Symmetry, and Thermodynamics, Phys. Rev. X 5, 021001 (2015).

[45] M. Lostaglio, D. Jennings, and T. Rudolph, Description of Quantum Coherence in Thermodynamic Processes Requires Constraints Beyond Free Energy, Nat. Commun. 6, 6383 (2015).

[46] K. Korzekwa, M. Lostaglio, J. Oppenheim, and D. Jennings, The Extraction of Work from Quantum Coherence, New J. Phys. 18, 023045 (2016).

[47] Similar arguments can be made for other states without full rank. For example, two copies of a rank-2 state in a fourdimensional system can be written as a pure state in a tensor product with a full-rank state. Such a resource therefore already contains a pure state, which can then be mapped to the ground state.

[48] F. Buscemi and G. Gour, Quantum Relative Lorenz Curves, Phys. Rev. A 95, 012110 (2017).

[49] G. Gour, Quantum Resource Theories in the Single-Shot Regime, Phys. Rev. A 95, 062314 (2017).

[50] C. Jarzynski, Nonequilibrium Equality for Free Energy Differences, Phys. Rev. Lett. 78, 2690 (1997).

[51] M. Tomamichel, Quantum Information Processing with Finite Resources, Springer Briefs in Mathematical Physics (Springer International Publishing AG, Heidelberg, 2016).

[52] A. Tari, The Specific Heat of Matter at Low Temperatures (Imperial College Press, London, 2003).

[53] Such a density of states is typical for many-body systems in the bulk of the energy, but deviations typically appear in the tails of the distribution.

[54] N. H. Y. Ng, L. Maninska, C. Cirstoiu, J. Eisert, and S. Wehner, Limits to Catalysis in Quantum Thermodynamics, New J. Phys. 17, 085004 (2015).

[55] W. D. Phillips, Nobel Lecture: Laser Cooling and Trapping of Neutral Atoms, Rev. Mod. Phys. 70, 721 (1998). 